\title{
Importance of centrifugal effects for the internal kink mode stability in toroidally rotating tokamak plasmas
}

\author{
C. Wahlberg, ${ }^{1}$ I. T. Chapman, ${ }^{2}$ and J. P. Graves ${ }^{3}$ \\ ${ }^{1}$ Department of Physics and Astronomy, EURATOM/VR Fusion Association, Uppsala University, \\ P.O. Box 516, SE-751 20 Uppsala, Sweden \\ ${ }^{2}$ EURATOM/CCFE Fusion Association, Culham Science Centre, Abingdon, Oxfordshire OX14 3DB, \\ United Kingdom \\ ${ }^{3}$ Centre de Recherches en Physique des Plasmas, Association EURATOM-Confederation Suisse, \\ EPFL, 1015 Lausanne, Switzerland
}

(Received 11 September 2009; accepted 16 October 2009; published online 25 November 2009)

\begin{abstract}
Analytical theory and two different magnetohydrodynamical stability codes are used in a study of the effects of toroidal plasma rotation on the stability of the ideal, internal kink mode in tokamaks. The focus of the paper is on the role that the centrifugal effects on the plasma equilibrium play for the stability of this mode, and results from one code where centrifugal effects are self-consistently included (CASTOR-FLOW) [E. Strumberger et al., Nucl. Fusion 45, 1156 (2005)] are compared with the results from another code where such effects are not taken into account (MISHKA-F) [I. T. Chapman et al., Phys. Plasmas 13, 062511 (2006)]. It is found that, even at rather modest flow speeds, the centrifugal effects are very important for the stability of the internal kink mode. While the results from the two codes can be quite similar for certain profiles in the plasma, completely opposite results are obtained for other profiles. A very good agreement between analytical theory and the numerical results are, both for inconsistent and consistent equilibria, found for plasmas with large aspect ratio. From the analytical theory, the distinctly different stability properties of equilibria with and without centrifugal effects included can be traced to the stabilizing effect of the geodesic acoustic mode (GAM) induced by the plasma rotation. This GAM exists solely as a consequence of the nonuniform plasma density and pressure created by the centrifugal force on the flux surfaces, and a stabilizing coupling of the internal kink instability to this mode cannot therefore take place if the centrifugal effects are not included in the equilibrium. In addition to the GAM stabilization, the effects of the radial profiles of the plasma density and rotation velocity are also found to be significant, and the importance of these effects increases with decreasing aspect ratio. (C) 2009 American Institute of Physics. [doi:10.1063/1.3263683]
\end{abstract}

\section{INTRODUCTION}

The internal kink instability with toroidal mode number $n=1$ and (dominating) poloidal mode number $m=1$ is, in one or another form, responsible for the sawtooth oscillations in tokamak plasmas where the safety factor $q$ drops below unity at the axis. ${ }^{1}$ In static, toroidal equilibria, the stability of the $m=n=1$ mode has been the subject of numerous analytical and numerical investigations, and most effects that are known to be of importance for the linear stability of this mode in such equilibria are today reasonably well understood. In many tokamaks heated by neutral beam injection (NBI), however, the resulting toroidal flows in the plasma have been found to lead to significant (and often stabilizing) effects on the sawtooth oscillations, presumably by strongly modifying the stability threshold of the underlying internal kink instability. Such effects on the sawteeth are seen especially in spherical tokamaks such as the Mega-Ampère Spherical Tokamak $(\mathrm{MAST})^{2}$ and the National Spherical Torus Experiment (NSTX), ${ }^{3}$ where toroidal plasma velocities exceeding the sound velocity can be produced by the neutral beams. In comparison with the extensive literature that exists on various aspects of the internal kink instability in static plasmas, very few investigations on the effects toroidal rotation of this order of magnitude on this instability have been published. Furthermore, existing results in this area are also not entirely consistent, especially in terms of the interpretation of the physical mechanism responsible for the stabilizing effect of the flow that is usually seen in these studies. $^{4-8}$ It is therefore of considerable interest to improve the understanding in this area. Better understanding of toroidal flow effects on the $m=n=1$ instability would be particularly valuable for the interpretation and prediction of the sawtooth activity in existing NBI heated tokamaks, especially in spherical tokamaks, but potentially useful also in connection with sawtooth control $^{9}$ in future tokamak experiments.

The well-known work by Waelbroeck in Ref. 4 is probably the first analysis of the effects of sonic, toroidal flows on the ideal $m=n=1$ instability in a toroidal plasma that takes the centrifugal effects on the plasma equilibrium into account. In this paper it was shown that, within the compressible, ideal magnetohydrodynamic (MHD) model, a rotation velocity of the order of the sound velocity at the $q=1$ radius has a strongly stabilizing effect on this instability, at least in plasmas with large aspect ratio. Furthermore, by the similarity of both the stability condition and the eigenfunction of the $m=n=1$ mode with a gyroscopically 
stabilized spinning top in gravitational field, the mechanism of the internal kink stabilization was interpreted as a gyroscopic effect. In addition, Waelbroeck showed that there are effects from the radial profiles of the density $\rho_{0}(r)$ and rotation frequency $\Omega(r)$ in the plasma that also have to be taken into account in the unstable regime of the mode. The same problem was a few years later addressed in a paper by Wahlberg and Bondeson, ${ }^{5}$ using an approach based on a computer-algebra expansion of the MHD stability (FriemanRotenberg) equation in the inverse aspect ratio $\varepsilon=r / R$ ( $r$ and $R$ denote the minor and major radius, respectively, in the plasma). Exactly the same result concerning the stabilizing effect of the rotation at the $q=1$ radius was found in that work, but a different interpretation of the responsible mechanism was suggested. Using an analogy from atmospheric physics, it was noted in Ref. 5 that the centrifugally created nonuniform plasma density and pressure on the $q=1 \mathrm{mag}$ netic surface, together with the "effective gravity" $\mathbf{g}=\Omega^{2} \mathbf{R}$ seen by the plasma in a corotating frame, represent a "stable stratification" of the plasma, with an associated oscillation frequency [or Brunt-Väisälä ${ }^{10}(\mathrm{BV})$ frequency] given by

$$
\omega_{\mathrm{BV}}^{2}=\frac{\mathcal{M}^{2} \Omega^{2}}{3}\left(1-\frac{1}{\Gamma}\right)
$$

Here, $\Gamma$ is the adiabatic index $(=5 / 3$ in collisional MHD), $\mathcal{M}=\left(\rho \Omega^{2} R_{0}^{2} / 2 p\right)^{1 / 2}$ is the sonic Mach number (apart from a factor of $\sqrt{5 / 6}$ ), and $p$ is the plasma pressure. The frequency in Eq. (1) appears in the stability problem, both in Ref. 4 and in Ref. 5, as a finite continuum frequency in the $q \approx 1$ layer, and it is this continuum frequency that, in a mathematical sense, is responsible for the stabilization of the kink mode, provided that $\Omega$ is sufficiently large at the radius where $q=1$. To leading order in $\varepsilon$, this stability condition was shown in Ref. 5 to be given by $\omega_{\mathrm{BV}}>\gamma_{B}$ where $\gamma_{B}$ is the static, or Bussac ${ }^{11}$ growth rate. Concerning the effect of the profiles of $\rho_{0}$ and $\Omega$ on the stability discussed in Ref. 4, this effect was not calculated in Ref. 5 since it represents a contribution to the potential energy $\delta W$ that formally is of higher order in $\varepsilon$ than the static, Bussac ${ }^{11}$ part of $\delta W$. As will be seen in many of the examples looked at in this paper, however, these higher-order profile effects are in practice very important at realistic values of $\varepsilon$, especially in equilibria where $\Delta q=1-q_{0}$ is small ( $q_{0}$ is the safety factor at the magnetic axis). Furthermore, the effect is included also in the equations derived in Ref. 5, even if this profile contribution to $\delta W$ never was explicitly calculated in that paper.

More recently, the problem of rotational stabilization of the internal kink mode has been investigated numerically by Chapman et al.,${ }^{6-8}$ using the MHD stability code MISHKA-F. ${ }^{6}$ This code treats the flow effects consistently in the stability equations but does not include any rotational (i.e., centrifugal) effects in the equilibrium. Ion diamagnetic drifts are, however, included in the code and this effect can explain the asymmetry between the co- and counter-NBI induced flow effects on the sawteeth in spherical tokamaks. Furthermore, for the profiles of density and rotation velocity used in these MISHKA-F calculations, stabilization of a similar nature as predicted in Refs. 4 and 5 was indeed seen, i.e., the growth rate of the internal kink instability going to zero at a sufficiently large rotation velocity. In view of the absence of centrifugal effects in the plasma equilibria used in these calculations, however, the applicability of the theories in Refs. 4 and 5 is not obvious, and an important question is to what extent the stabilization seen in these studies in spite of this can be understood in terms of the stabilizing mechanisms discussed in Refs. 4 and 5. It is, for instance, quite clear that due to the uniform plasma density and pressure on the flux surfaces of the equilibria used, the basis for the BV mechanism discussed in Ref. 5 vanishes, and stabilization by this effect is therefore not possible. On the other hand, since the "shift-tilt" $m=n=1$ eigenmode ${ }^{4}$ also in the MISHKA-F calculations sit on top of a rotating plasma torus with finite angular momentum, some gyroscopic, stabilizing effect from the rotation should be present in these calculations, in spite of the absence of centrifugal effects in the equilibrium (note that gyroscopic stabilization of a spinning top does not require that the rotation changes the "equilibrium" of the top). A highly interesting question concerning the results in Refs. $6-8$ is then to what extent the stabilization seen there comes from a finite value of the continuum frequency in Eq. (1) (i.e., if it is related to the stabilization that is called gyroscopic in Ref. 4). Hypothetically one could, for instance, imagine the continuum frequency in Eq. (1) to be related to the rotation alone, without the critical dependence on the density and pressure distribution on the flux surfaces discussed in Ref. 5. If this was the case, stabilization due to a finite continuum frequency would occur even without any centrifugal effects included in the equilibrium, for instance in the equilibria used in the MISHKA-F computations. A more likely explanation, however, is that the stabilization seen numerically has more to do with the rotation-dependent part of the potential energy of the internal kink mode derived in Ref. 4, which is an effect that does not depend on centrifugal effects and is entirely different from the stabilization by the continuum frequency.

In order to resolve this issue, the stability analysis in Ref. 5 will be repeated in the present paper, using a plasma equilibrium parametrized in such a way that the effects of the equilibrium terms that have to do with the centrifugal force can be traced through the computer-algebra calculation. More specifically, we express the equilibrium profiles of the pressure $p$, density $\rho$, and the poloidal beta value $\beta_{p}$ of the plasma in the form

$$
\begin{aligned}
& \frac{p(r, \theta)}{p_{0}(r)}=\frac{\rho(r, \theta)}{\rho_{0}(r)}=\exp \left[\frac{\sigma_{1} \rho \Omega^{2}\left(R^{2}-R_{0}^{2}\right)}{2 p}\right], \\
& \beta_{p}=-\frac{2 \mu_{0} R_{0}^{2} q^{2}}{B_{0}^{2} r^{4}} \int_{0}^{r} r^{\prime 2} \frac{d}{d r^{\prime}}\left(p_{0}+\sigma_{2} \mathcal{M}^{2} p_{0}\right) d r^{\prime},
\end{aligned}
$$

respectively. These equations replace Eqs. (5) and (13), respectively, in Ref. 5. Here, $R_{0}, B_{0}$, and $\mu_{0}$ denote the major 
radius of the plasma center, the toroidal magnetic field, and the vacuum permeability, respectively. Furthermore, $(r, \theta, \varphi)$ is the flux coordinate system defined in Ref. 5 and Eq. (2a) expresses the fact that, in a rotating plasma, the density and pressure are no longer flux functions but vary with $\theta$ on the flux surfaces. The correct equilibrium relations are obtained for $\sigma_{1}=\sigma_{2}=1$, and other values of $\sigma_{1}$ and $\sigma_{2}$ lead to a nonself-consistent stability problem. Especially, we can switch off the centrifugally induced nonuniformity of the density and pressure on the flux surfaces by choosing $\sigma_{1}=0$, whereas for $\sigma_{2}=0$ we remove the part of the Shafranov shift that is caused by the centrifugal force [the Shafranov shift is given by $\Delta^{\prime}=-\left(r / R_{0}\right)\left(\beta_{p}+\ell_{i} / 2\right)$ as usual, ${ }^{4,5}$ where $\ell_{i}$ is the internal inductance and $\beta_{p}$ is given by Eq. (2b)].

Although an analytical study based on Eq. (2) might be sufficient to identify the origin of the stabilizing continuum frequency in Eq. (1), and also the effect of the enhanced Shafranov shift, conclusions drawn on the basis of analytical theory alone would be subject to great uncertainty both because of the rather complex (and nonself-consistent) nature of the problem, and also due to the $\varepsilon \ll 1$ approximation (as well as other assumptions) used in the analysis. As a major extension compared with previous work, growth rates will therefore be calculated also numerically in the present paper, using the two stability codes MISHKA-F (Ref. 6) and CASTOR-FLOW. ${ }^{12}$ The latter code includes, in contrast to MISHKA-F, the centrifugal effects from the plasma rotation in the equilibrium. Since both of these codes have been derived from the CASTOR ideal MHD stability code, ${ }^{13,14}$ any differences observed are resultant from the treatment of the equilibrium flow rather than from the numerical formulation of the eigenvalue solvers. Hence, it will be meaningful and of interest to compare, first of all, results from these two codes to each other, and thereafter also the analytical growth rates in the consistent case $\sigma_{1}=\sigma_{2}=1$ with the CASTOR-FLOW results and the corresponding analytical results in the inconsistent case $\sigma_{1}=\sigma_{2}=0$ with the growth rates obtained with MISHKA-F (with the ion diamagnetic terms switched off).

Figure 1 illustrates the striking difference that can be obtained between the consistent and inconsistent treatments for a particular choice of equilibrium profiles. The upper curves show the analytical (dashed) and numerical (solid) growth rates in the inconsistent case as functions of the rotation velocity at the axis in a plasma with $\varepsilon_{a}=a / R_{0}=0.1$ ( $a$ is the minor radius of the plasma), a parabolic $q$-profile with $q_{0}=0.938, r_{1} / a=0.3\left(r_{1}\right.$ is the radius where $\left.q=1\right)$ and $\beta_{p 0}=0.3$ [with $\beta_{p 0}$ referring to the static part of Eq. (2b)]. The corresponding results in the consistent case are shown by the lower curves. The analytical growth rates in the figure are calculated from Eq. (20), and are, both for inconsistent and consistent equilibria, seen to be in good agreement with the numerical growth rates. Furthermore, for the parabolic profiles of the density and rotation used in this plot, $\rho_{0}(r) / \rho_{0}(0)=\Omega(r) / \Omega(0)=1-r^{2} / a^{2}$, the flow is seen to have a destabilizing effect on the internal kink mode when $\sigma_{1}=\sigma_{2}$ $=0$ whereas in the consistent case, $\sigma_{1}=\sigma_{2}=1$, the flow is instead found to be strongly stabilizing. It will be shown in this paper that the absence of rotational stabilization in the

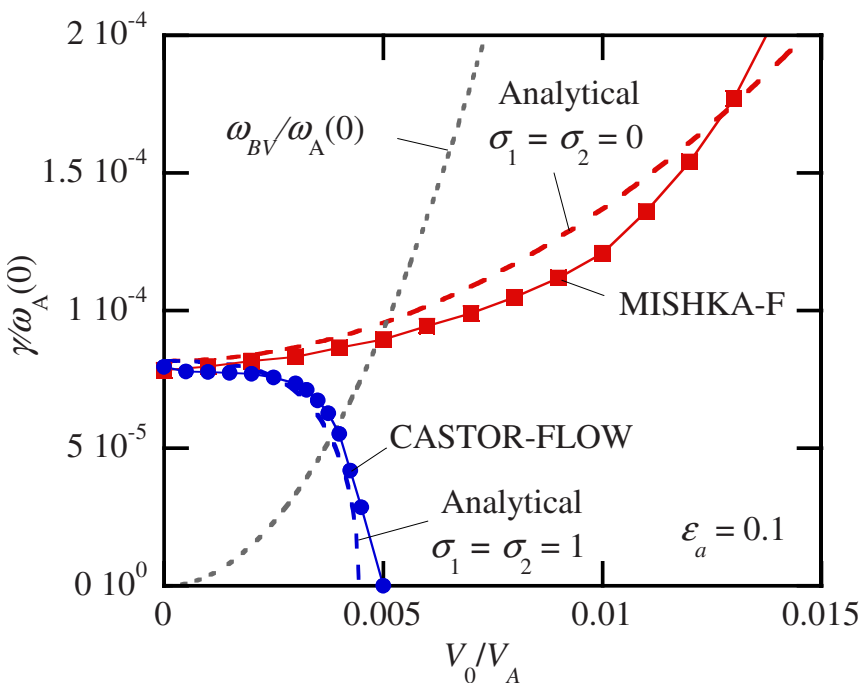

FIG. 1. (Color online) Growth rate vs rotation velocity at the axis calculated for a self-consistent equilibrium $\left(\sigma_{1}=\sigma_{2}=1\right)$ and for a nonself-consistent equilibrium $\left(\sigma_{1}=\sigma_{2}=0\right)$. The dashed curves show the analytical predictions from Eq. (20) while the symbols give the numerical results obtained with the two different numerical codes indicated. The plasma parameters are $\varepsilon_{a}=0.1, q_{0}=0.938, r_{1} / a=0.3, \beta_{p 0}=0.3$, and $\beta_{0}=0.66 \%$ and the density and rotation profiles are both parabolic. The dotted curve shows the BV frequency in Eq. (1), and stabilization in the consistent case is seen to occur approximately when this frequency exceeds the static growth rate.

inconsistent case mainly is a consequence of the fact that the continuum frequency in Eq. (1) turns out to vanish when $\sigma_{1}=0$. However, both of the effects expressed by $\sigma_{1}=1$ and $\sigma_{2}=1$ in Eqs. (2a) and (2b), respectively, contribute to the stabilization seen in the lower curves in the figure. Furthermore, by plotting also the BV frequency in Eq. (1) in the same diagram (shown by the dotted curve) it is seen that the stabilization in the consistent case occurs approximately when $\omega_{\mathrm{BV}}$ exceeds the static growth rate $\gamma_{B}$, as predicted in Ref. 5. We point out, however, that the validity of this stability condition is due to the small value of $\varepsilon_{a}$ used in Fig. 1, and is in general not applicable in plasmas with realistic values of $\varepsilon_{a}$.

In a recent paper, ${ }^{15}$ the effect of the parametrization in Eq. (2a) for the continuous MHD spectrum of rotating plasmas was discussed, in particular the vanishing of the stabilizing continuum frequency in Eq. (1) as $\sigma_{1} \rightarrow 0$. It was also shown that this continuum frequency actually represents a second branch of the geodesic acoustic mode $(\mathrm{GAM}),{ }^{16}$ induced by the rotation of the plasma. ${ }^{17,18}$ The stabilization of the $m=n=1$ instability by toroidal rotation (for $\sigma_{1} \neq 0$ ) can therefore also be described in terms of a coupling of this mode to the rotation-induced GAM, and this terminology will generally be used in the rest of this paper.

It should be pointed out that while the inconsistent and consistent models predict very different growth rates for the particular equilibrium studied in Fig. 1, other choices of the radial profiles of the density and/or rotation velocity can lead to quite similar results in the inconsistent and consistent cases. Indeed, in all the MAST equilibria analyzed in Ref. 7, the critical rotation required for stabilization of the internal kink mode never differs by more than $20 \%$ between the con- 
sistent (CASTOR-FLOW) and inconsistent (MISHKA-F) treatments. Furthermore, by the absence of the stabilizing GAM coupling in the inconsistent model, MISHKA-F generally overestimates and gives an upper bound on the rotation velocity required to stabilize the mode. These aspects, and also the sensitivity of the mode stability to the profiles of the density and rotation velocity, are discussed in more detail in a separate paper. ${ }^{19}$

The rest of the present paper is organized as follows. In the next section, the details of the analysis of the $m=n=1$ stability problem using the parametrized equilibrium in Eq. (2) are presented. Thereafter, in Sec. III, analytical growth rates both in the inconsistent $\sigma_{1}=\sigma_{2}=0$ and the consistent $\sigma_{1}=\sigma_{2}=1$ cases are calculated and compared with numerical computations using MISHKA-F and CASTOR-FLOW, respectively. In Sec. IV, numerical $n=1$ growth rates versus rotation velocity for experimentally relevant profiles from MAST and for a typical conventional aspect ratio tokamak are calculated, in both cases showing the importance of using equilibria with centrifugal effects included. The conclusions of the paper are given in Sec. V.

\section{STABILITY ANALYSIS}

We replace the equilibrium relations in Eqs. (5) and (13) in Ref. 5 with Eqs. (2a) and (2b), respectively, and repeat the computer algebra derivation of the stability equations in that paper. We keep all other expressions involving $\Omega$ unchanged in the derivation, which is based on the Frieman-Rotenberg ${ }^{20}$ eigenvalue equation for the Lagrangian perturbation $\xi \sim e^{-i \omega t}$ in a flowing plasma:

$$
\begin{gathered}
\rho \omega^{2} \xi+2 i \rho \omega \mathbf{v} \cdot \nabla \xi-\rho \mathbf{v} \cdot \nabla[(\mathbf{v} \cdot \nabla) \xi]+\nabla \cdot[\rho \xi(\mathbf{v} \cdot \nabla) \mathbf{v}] \\
-\nabla \delta P+(\mathbf{B} \cdot \nabla) \mathbf{Q} / \mu_{0}+(\mathbf{Q} \cdot \nabla) \mathbf{B} / \mu_{0}=0
\end{gathered}
$$

$$
\delta P=-\xi \cdot \nabla p-\Gamma p \nabla \cdot \xi+\mathbf{B} \cdot \mathbf{Q} / \mu_{0},
$$

$$
\mathbf{Q}=\nabla \times(\xi \times \mathbf{B}) .
$$

Here, $\mathbf{B}$ and $\mathbf{v}$ denote the equilibrium magnetic field and velocity, respectively, $\delta P$ the perturbed, total pressure, and $\mathbf{Q}$ the perturbed magnetic field. Furthermore, $\mathbf{v}=\Omega(r) \mathbf{e}_{\varphi}$ where $\mathbf{e}_{\varphi}=R \hat{\varphi}$ is a covariant basis vector of the flux coordinates $(r, \theta, \varphi)$. The analysis in Ref. 5 as well as in the present paper leads to a system of equations describing the coupling of the $(m, n)=(1,1)$ and $(m, n)=(2,1)$ Fourier components $\xi_{1}$ and $\xi_{2}$ of the radial component $\xi^{r}$ of $\xi$. For $\sigma_{1} \neq 1$ and $\sigma_{2} \neq 1$, the following modifications of the final stability equations in Ref. 5, Eqs. (38a) and (38b), are obtained. First, a new term that has to do with the modified Shafranov shift appears in the equation for the $m=2$ sideband:

$$
\begin{aligned}
\mathcal{L}_{2} \xi_{2} & +\frac{d}{d r}\left(r^{3} T_{1} \frac{d \xi_{1}}{d r}\right)+r^{2} T_{2} \frac{d \xi_{1}}{d r} \\
- & \frac{\left(\sigma_{2}-1\right) \mu_{0} R_{0}^{3} r^{3}}{B_{0}^{2}} \frac{d}{d r}\left[r^{-1}\left(\rho_{0} \Omega^{2}\right)^{\prime} \xi_{1}\right]=0 .
\end{aligned}
$$

The operator $\mathcal{L}_{2}$ above is defined by

$$
\mathcal{L}_{m} \equiv \frac{d}{d r}\left[r^{3}(m \mu-1)^{2} \frac{d}{d r}\right]-r\left(m^{2}-1\right)(m \mu-1)^{2},
$$

where $\mu \equiv 1 / q$ is the inverse safety factor. Furthermore, the coefficients $T_{1}$ and $T_{2}$ in Eq. (4) are unchanged, i.e.,

$$
\begin{aligned}
& T_{1}=\left(-6 \mu^{2}+6 \mu-1\right) \Delta^{\prime}+\left(-4 \mu^{2}+10 \mu-\frac{11}{2}\right) \frac{r}{R_{0}}, \\
& T_{2}=\left(6 \mu^{2}-6 \mu+3\right) \Delta^{\prime}+\left(2 \mu^{2}-4 \mu+\frac{7}{2}\right) \frac{r}{R_{0}},
\end{aligned}
$$

but an indirect effect of $\sigma_{2}$ appears in these coefficients via the modified Shafranov shift $\Delta^{\prime}=-\left(r / R_{0}\right)\left(\beta_{p}+\ell_{i} / 2\right)$, with $\beta_{p}$ given by Eq. (2b). Second, several new terms appear in the final $m=1$ equation, Eq. (38a) in Ref. 5. Since all these extra terms involve $\rho_{0} \Omega^{2}$ in one or another form, we include them in the kinetic energy term $\mathcal{T}_{1}$. So, before we specify $\mathcal{T}_{1}$, the modified $m=1$ equation is identical to Eq. (38a) in Ref. 5, i.e.,

$$
\begin{aligned}
\mathcal{L}_{1} \xi_{1} & +\mathcal{T}_{1}\left(\xi_{1}, \xi_{2}\right)+W_{1} \xi_{1}+\frac{d}{d r}\left(r^{3} W_{2} \frac{d \xi_{1}}{d r}\right)+r^{2} W_{3} \frac{d \xi_{1}}{d r} \\
+ & \frac{d}{d r}\left(r^{3} W_{4} \frac{d \xi_{2}}{d r}\right)+\frac{d}{d r}\left(r^{2} W_{5} \xi_{2}\right)=0,
\end{aligned}
$$

with $W_{1}-W_{5}$ also unchanged, i.e.,

$$
\begin{aligned}
W_{1}= & \frac{d}{d r}\left[\left(-\frac{3}{2} \mu^{2}+\frac{3}{2} \mu-\frac{3}{4}\right) \frac{\Delta^{\prime} r^{3}}{R_{0}}-\frac{1}{2}(\mu-1)^{2} \frac{r^{4}}{R_{0}^{2}}\right] \\
& -\left(\frac{1}{2} \mu^{2}+\frac{1}{4}\right) \frac{r^{3}}{R_{0}^{2}} \\
W_{2}= & \left(2 \mu^{2}-2 \mu+\frac{1}{4}\right) \Delta^{\prime 2}+\left(-\mu^{2}+\frac{1}{2} \mu+\frac{1}{4}\right) \frac{\Delta^{\prime} r}{R_{0}} \\
& +\left(-\frac{1}{4} \mu^{2}+\frac{3}{4} \mu-\frac{7}{16}\right) \frac{r^{2}}{R_{0}^{2}}, \\
W_{3}= & -3(\mu-1)^{2} \frac{\Delta^{\prime} r}{R_{0}},
\end{aligned}
$$




$$
\begin{aligned}
& W_{4}=\left(-\frac{3}{2} \mu^{2}+\frac{3}{2} \mu-\frac{1}{4}\right) \Delta^{\prime}+\left(-\frac{1}{2} \mu^{2}+\frac{3}{2} \mu-\frac{7}{8}\right) \frac{r}{R_{0}} \\
& W_{5}=\left(-\frac{3}{2} \mu^{2}+\frac{3}{2} \mu-\frac{3}{4}\right) \Delta^{\prime}-\frac{3 r}{8 R_{0}} .
\end{aligned}
$$

The indirect effect of $\sigma_{2}$ from $\Delta^{\prime}$ should be included also in these terms. The inertia term $\mathcal{T}_{1}\left(\xi_{1}, \xi_{2}\right)$ includes several $\sigma_{1}$, $\sigma_{2}$-dependent terms. In the self-consistent case $\sigma_{1}=\sigma_{2}=1$ it turned out to be possible to write $\mathcal{T}_{1}$ in the relatively simple form of Eqs. (36) and (37) in Ref. 5. Such a simple form of $\mathcal{T}_{1}$ does not seem to be possible here, but one can nevertheless arrange the terms in the following somewhat similar form:

$$
\begin{aligned}
\mathcal{T}_{1}\left(\xi_{1}, \xi_{2}\right)= & \frac{d}{d r}\left(r^{3} A_{1} \frac{d \xi_{1}}{d r}\right)+r^{2} \frac{d A_{2}}{d r} \xi_{1}+r^{3} A_{3} \frac{d \xi_{1}}{d r} \\
& -\frac{\left(\sigma_{2}-1\right) \mu_{0} R_{0}^{2} r^{2}(2 \mu-5)}{4 B_{0}^{2}}\left(\rho_{0} \Omega^{2}\right)^{\prime} \xi_{1} \\
& +\frac{\left(\sigma_{2}-1\right) \mu_{0} R_{0}^{3}}{B_{0}^{2}}\left\{\frac{1}{2} \frac{d}{d r}\left[r^{2}\left(\rho_{0} \Omega^{2}\right)^{\prime} \xi_{2}\right]\right. \\
& \left.-\frac{1}{8} \frac{d}{d r}\left[r^{2}\left(\rho_{0} \Omega^{2}\right)^{\prime}\left((4 \mu-2) \Delta^{\prime}-\frac{r}{R_{0}}\right) \xi_{1}\right]\right\} .
\end{aligned}
$$

A major effect from the rotation on the mode stability comes from the coefficient $A_{1}$, which is now given by

$$
\begin{aligned}
A_{1}= & -\frac{\omega_{D}^{2}-\sigma_{1} \Omega^{2} \mathcal{M}^{2}}{\omega_{A}^{2}}-\frac{\left(\alpha_{1} \omega_{D}^{2}-\alpha_{2} \omega_{D} \Omega+\alpha_{3} \Omega^{2}\right) \Omega^{2}+\left(\omega_{D}^{2}-2 \omega_{D} \Omega\right) \omega_{s}^{2}}{\omega_{A}^{2}\left(\omega_{s}^{2}-\omega_{D}^{2}\right)} \\
& -\frac{\left[\alpha_{1} \omega_{D}^{2}+\alpha_{2}(2 \mu-1) \omega_{D} \Omega+\alpha_{3}(2 \mu-1)^{2} \Omega^{2}\right] \Omega^{2}+\left[\omega_{D}^{2}+2(2 \mu-1) \omega_{D} \Omega\right] \omega_{s}^{2}}{\omega_{A}^{2}\left[(2 \mu-1)^{2} \omega_{s}^{2}-\omega_{D}^{2}\right]},
\end{aligned}
$$

where $\alpha_{1}=\left(\sigma_{1}+3\right) / 2, \alpha_{2}=\left(\sigma_{1}+1\right) / 2$, and $\alpha_{3}=\sigma_{1} / 4$. Furthermore, $\omega_{A}^{2}=B_{0}^{2} /\left(\mu_{0} \rho_{0} R_{0}^{2}\right), \omega_{s}^{2}=\Gamma p_{0} /\left(\rho_{0} R_{0}^{2}\right), \omega_{D} \equiv \omega+\Omega$, and the expression for $A_{1}$ has been made dimensionally correct (i.e., dimensionless) in the present paper by introducing the quantity $B_{0}^{2} / \mu_{0}$ [also in Eqs. (2b), (4), and (9)], a quantity that was normalized to unity in Ref. 5. Note that $A_{1}$ depends on $\sigma_{1}$ and not on $\sigma_{2}$, whereas the other terms in Eq. (9) (apart from $A_{2}$ and $A_{3}$, discussed later) depend on $\sigma_{2}$ but not on $\sigma_{1}$. When $\sigma_{1}=1$, Eq. (10) is seen to reproduce Eq. (37a) in Ref. 5.

From Eqs. (4), (5), (6a), (6b), (7), (8a)-(8e), (9), and (10) we can now perform a similar stability analysis as in Sec. IV in Ref. 5. As the first step it is convenient to identify the roots of $A_{1}=0$ when $q=1$. This equation becomes

$$
\begin{gathered}
\left(\frac{\omega_{D}}{\omega_{s}}\right)^{4}-\left(\frac{\omega_{D}}{\omega_{s}}\right)^{2}\left(3+\frac{6+2 \sigma_{1}}{\Gamma} \mathcal{M}^{2}+\frac{2 \sigma_{1}}{\Gamma} \mathcal{M}^{4}\right) \\
+\frac{2 \sigma_{1}}{\Gamma} \mathcal{M}^{4}\left(1-\frac{1}{\Gamma}\right)=0,
\end{gathered}
$$

where $\mathcal{M}$ now stands for the Mach number at $q=1$. For $\mathcal{M} \sim 1$, we obtain the two roots

$$
\begin{aligned}
\left(\frac{\omega_{D}}{\omega_{s}}\right)^{2}= & \left(\frac{3}{2}+\frac{\mathcal{M}^{2}\left(\sigma_{1} \mathcal{M}^{2}+3+\sigma_{1}\right)}{\Gamma}\right) \\
& \pm\left[\left(\frac{3}{2}+\frac{\mathcal{M}^{2}\left(\sigma_{1} \mathcal{M}^{2}+3+\sigma_{1}\right)}{\Gamma}\right)^{2}\right. \\
& \left.-\frac{2 \sigma_{1}}{\Gamma} \mathcal{M}^{4}\left(1-\frac{1}{\Gamma}\right)\right]^{1 / 2} .
\end{aligned}
$$

Assuming that $\mathcal{M}^{2} \ll 1$ and using the same notation as in Ref. 15 , these two roots are given by

$$
\begin{aligned}
& \omega_{D}^{2}=\omega_{\mathrm{GAM} 1}^{2}=\omega_{s}^{2}\left(3+\frac{\left(6+2 \sigma_{1}\right) \mathcal{M}^{2}}{\Gamma}+\cdots\right), \\
& \omega_{D}^{2}=\omega_{\mathrm{GAM} 2}^{2}=\omega_{\mathrm{BV}}^{2}=\frac{\sigma_{1} \mathcal{M}^{2} \Omega^{2}}{3}\left(1-\frac{1}{\Gamma}\right)+\cdots .
\end{aligned}
$$

$A_{1}$ at the $q=1$ radius can now be expressed in the form

$$
A_{1}\left(r_{1}\right)=\frac{\left(\omega_{D}^{2}-\omega_{\mathrm{GAM} 1}^{2}\right)\left(\omega_{D}^{2}-\omega_{\mathrm{GAM} 2}^{2}\right)}{\omega_{A}^{2}\left(\omega_{s}^{2}-\omega_{D}^{2}\right)} \approx \frac{F(\mathcal{M}) \gamma_{0}^{2}}{\omega_{A}^{2}}
$$

where $\left|\omega_{D}^{2}\right| \ll \omega_{s}^{2} \sim \omega_{\mathrm{GAM} 1}^{2}$ has been assumed, $\quad F(\mathcal{M})$ $=\omega_{\mathrm{GAM} 1}^{2} / \omega_{s}^{2}=3+\left(6+2 \sigma_{1}\right) \mathcal{M}^{2} / \Gamma+\ldots$ and $\gamma_{0}^{2}=\omega_{\mathrm{GAM} 2}^{2}-\omega_{D}^{2}$. These two quantities play a similar role here as $\nu_{0}$ and the matching parameter $\gamma_{0}$ play in Waelbroeck's analysis. ${ }^{4}$ 
As the next step we integrate Eq. (7) from $r=0$ up to the layer $r=r_{1}$. As usual, we assume a (zeroth order) "top hat" form of the eigenfunction $\xi_{1}$, i.e., $\xi_{1}=\hat{\xi}$ for $0 \leq r<r_{1}$, $\xi_{1} \equiv 0$ for $r>r_{1}$ and, for $r \approx r_{1}$, we introduce the layer variable $x=\left(r-r_{1}\right) / \varepsilon^{2}$. Equation (7) is then transformed to

$$
\begin{aligned}
\frac{1}{\varepsilon^{2}}\left[\varepsilon^{4}\left(q^{\prime}\right)^{2} x^{2}+\frac{F(\mathcal{M}) \gamma_{0}^{2}}{\omega_{A}^{2}}\right] r_{1}^{3} \frac{d \xi_{1}}{d x}+r_{1}^{3} A_{3}\left(r_{1}\right) \xi_{1}(x) \\
=-\hat{\xi} \int_{0}^{r_{1}} r^{2} \frac{d A_{2}}{d r} d r+\frac{\left(\sigma_{2}-1\right) \mu_{0} R_{0}^{2}}{4 B_{0}^{2}} \hat{\xi} \int_{0}^{r_{1}} r^{2}(2 \mu-5)\left(\rho_{0} \Omega^{2}\right)^{\prime} d r-\frac{\left(\sigma_{2}-1\right) \mu_{0} R_{0}^{3}}{B_{0}^{2}} \\
\quad \times\left\{\frac{1}{2}\left[r^{2}\left(\rho_{0} \Omega^{2}\right)^{\prime} \xi_{2}\right]_{r=r_{1}}-\frac{1}{8}\left[r^{2}\left(\rho_{0} \Omega^{2}\right)^{\prime}\left((4 \mu-2) \Delta^{\prime}-\frac{r}{R_{0}}\right) \xi_{1}\right]_{r=r_{1}}\right\} \\
-\hat{\xi} \int_{0}^{r_{1}} W_{1} d r-\left.r_{1}^{3} W_{2} \frac{d \xi_{1}}{d r}\right|_{r=r_{1}}-\left.r_{1}^{3} W_{4} \frac{d \xi_{2}}{d r}\right|_{r=r_{1}}-\left.r_{1}^{2} W_{5} \xi_{2}\right|_{r=r_{1} .} .
\end{aligned}
$$

It can be shown, using a similar analysis as in Eq. (41) in Ref. 5 that the right hand side of this equation is free from singularities and is continuous at $r=r_{1}$. The problem is therefore well-formulated and solvable also for the nonselfconsistent equilibrium in Eq. (2).

To proceed further we express the right hand side of Eq. (15) in the form $\hat{\xi} r_{1}^{4} \delta \hat{W} / R_{0}^{2}$, where

$$
\delta \hat{W}=\delta \hat{W}_{\mathrm{Bussac}}+\delta \hat{W}_{\mathrm{Rot} 1}+\delta \hat{W}_{\mathrm{Rot} 2}
$$

Here $\delta \hat{W}_{\text {Bussac }}$ is the usual Bussac form of the (normalized) internal kink potential energy, ${ }^{11}$ although with $\beta_{p}$ modified by the rotation in accordance with Eq. (2b). This part of $\delta \hat{W}$ comes from the four last terms in Eq. (15), but it does not include the part of $\xi_{2}$ caused by the $\sigma_{2}-1$ term in Eq. (4). The term $\delta \hat{W}_{\text {Rot1 }}$ represents the contribution from the integral including $d A_{2} / d r$ in Eq. (15), whereas $\delta \hat{W}_{\text {Rot2 }}$ represents all terms in Eq. (15) proportional to $\sigma_{2}-1$, including the $\sigma_{2}-1$ dependent part of $\xi_{2}$ from Eq. (4). The full expressions for these three contributions to $\delta \hat{W}$ in Eq. (16) will be given in the following subsections.

The coefficient $A_{3}$ in Eq. (15) is given by

$$
A_{3}\left(r_{1}\right)=\frac{\left(\sigma_{1}-1\right) \Omega \omega_{D}}{\omega_{s}^{2}-\omega_{D}^{2}}\left(\frac{\Omega^{2}}{\omega_{A}^{2}}\right)^{\prime} .
$$

In the unstable range of the mode it turns out that $\omega_{D} \sim \gamma_{0}$ $\sim \varepsilon^{2} \omega_{A} \sim \varepsilon \omega_{s}$. Furthermore, since $\Omega \sim \omega_{s}$ (or smaller) it is seen that the $A_{3}\left(r_{1}\right) \xi_{1}(x)$ term in Eq. (15) becomes at least a factor $\varepsilon$ smaller than the $d \xi_{1} / d x$ terms and can therefore be neglected. By matching the solution of the layer Eq. (15) to the "top hat," outer solution as $x \rightarrow \pm \infty$ we obtain (assuming that $\delta \hat{W}$ is real and negative),

$$
\frac{\gamma_{0}}{\omega_{A}(0)}=-\frac{\pi \delta \hat{W}}{s_{1} \sqrt{3 F_{\rho}}}\left(\frac{r_{1}}{R_{0}}\right)^{2},
$$

where $s_{1}=r_{1} q^{\prime}\left(r_{1}\right)$ and $F(\mathcal{M})$ has been approximated with 3 (assuming that $\mathcal{M}^{2} / \Gamma \ll 1$ ). Furthermore, for the comparison with numerical growth rates in the next section, the extra factor $F_{\rho}=\rho_{0}\left(r_{1}\right) / \rho_{0}(0)$ in Eq. (18) normalizes $\gamma_{0}$ to $\omega_{A}(0)$ rather than to $\omega_{A}\left(r_{1}\right)$.

Finally, we express the real growth rate $\gamma$ of the mode in terms of $\gamma_{0}$ in Eq. (18) and $\omega_{\mathrm{GAM} 2}$ in Eq. (13b), using the relation $\gamma_{0}^{2}=\omega_{\mathrm{GAM} 2}^{2}-\omega_{D}^{2}$ introduced in Eq. (14). Notice that $\omega_{D}$ in this relation refers to $\omega_{D}\left(r_{1}\right)=\omega_{r}+i \gamma+\Omega\left(r_{1}\right)$. It is seen that, since both $\gamma_{0}^{2}$ and $\omega_{\mathrm{GAM} 2}^{2}$ are real, and $\gamma_{0}^{2}>\omega_{\mathrm{GAM} 2}^{2}$ for sufficiently low rotation frequencies (lower than a possible, stabilizing rotation frequency) $-\omega_{D}^{2}$ is also real and positive in the unstable regime of the mode. It follows that $\omega_{r}=-\Omega\left(r_{1}\right)$ and

$$
\gamma^{2}=\gamma_{0}^{2}-\omega_{\mathrm{GAM} 2}^{2} \text {. }
$$

If we now call $\hat{\Omega}=\Omega(0) / \omega_{A}(0)$ (denoted by $V_{0} / V_{A}$ in the figures $), \quad \beta_{0}=2 \mu_{0} p_{0}(0) / B_{0}^{2}, \quad F_{p}=p_{0}\left(r_{1}\right) / p_{0}(0), \quad F_{\Omega}=\Omega\left(r_{1}\right) /$ $\Omega(0), \varepsilon_{1}=r_{1} / R_{0}$, and set $\Gamma=5 / 3$, we obtain the growth rate in the final form

$$
\frac{\gamma}{\omega_{A}(0)}=\left(\frac{\pi^{2} \delta \hat{W}^{2} \varepsilon_{1}^{4}}{3 F_{\rho} s_{1}^{2}}-\frac{2 \sigma_{1} F_{\rho} F_{\Omega}^{4} \hat{\Omega}^{4}}{15 F_{p} \beta_{0}}\right)^{1 / 2},
$$

with the potential energy $\delta \hat{W}$ given by Eq. (16). In the following three subsections, we look more closely at the three different parts of $\delta \hat{W}$.

\section{A. Expression for $\delta \hat{W}_{\text {Bussac }}$}

The Bussac part of $\delta \hat{W}$ originates from the terms involving $W_{1}-W_{5}$ in Eq. (15) and can be expressed in the wellknown form, ${ }^{11,21,22}$ 


$$
\begin{aligned}
\delta \hat{W}_{\text {Bussac }}= & \frac{32(b-c) \sigma+9(b-1)(1-c)}{64(b-c)} \\
& -\frac{3(b-1)(c+3)}{8(b-c)}\left(\beta_{p}+\sigma\right) \\
& -\frac{(b+3)(c+3)}{4(b-c)}\left(\beta_{p}+\sigma\right)^{2},
\end{aligned}
$$

where

$$
\sigma=\frac{\ell_{i}}{2}-\frac{1}{4}=\int_{0}^{r_{1}} \frac{d r}{r_{1}}\left(\frac{r}{r_{1}}\right)^{3}\left(\frac{1}{q^{2}}-1\right),
$$

and $b$ and $c$ are determined from the solution of the Euler equation $\mathcal{L}_{2} \xi_{2}=0$, with

$$
b=\left.\frac{r}{\xi_{2}} \frac{d \xi_{2}}{d r}\right|_{r_{1}-0} \text { and } c=\left.\frac{r}{\xi_{2}} \frac{d \xi_{2}}{d r}\right|_{r_{1}+0} .
$$

Thus, $b$ is determined from the solution in the inner region $0 \leq r \leq r_{1}$ where the boundary condition $\xi_{2}(r=0)=0$ applies, whereas $c$ is determined by the solution in the outer region $r \geq r_{1}$, where the boundary condition $d \xi_{2} / d r\left(r=r_{2}\right)=0$ should be used. Here $r_{2}$ is the radius where $q=2$. If $r_{2}$ does not exist in the plasma, the boundary condition for $\xi_{2}$ is instead $\xi_{2}(r=a)=0$, where $a$ is the plasma radius.

In the comparison of the analytical growth rates given by Eq. (20) with numerical results in the next section, a $q$-profile of the form

$$
q(r)=1-\Delta q\left[1-\left(\frac{r}{r_{1}}\right)^{\lambda}\right]
$$

with $\lambda=2$ and a rather small $\Delta q=1-q_{0}$ will be used in many of the examples. In this case, the following asymptotic expansion of $\delta \hat{W}_{\text {Bussac }}$ is valid, ${ }^{22}$

$$
\begin{aligned}
\delta \hat{W}_{\text {Bussac }}= & \Delta q\left(\frac{13}{48}-3 \beta_{p}^{2}\right)+(\Delta q)^{2} \\
& \times\left\{\frac{5}{32}-\frac{5 \beta_{p}}{2}+6[1+\ln (\Delta q)] \beta_{p}^{2}\right\}+\cdots .
\end{aligned}
$$

The error in $\delta \hat{W}_{\text {Bussac }}$ obtained by using Eq. (25) instead of the full form in Eq. (21) is, in the examples looked at here, typically a few percent and always less than $12 \%$. In the cases where this approximation is used in the next section, the value of $\delta \hat{W}_{\text {Bussac }}$ from Eq. (25) has been multiplied with a correction factor of order $f_{\text {corr }}=1 \pm 0.12$ in order to normalize the growth rate from Eq. (20) to the numerical, static growth rate.

If $\Delta q$ is not small (and $\lambda$ not necessarily 2), one can instead use the following approximations of the quantities $\sigma$, $b$, and $c$ in Eqs. (22) and (23), which gives a very accurate value of $\delta \hat{W}_{\text {Bussac }}$ in Eq. (21), ${ }^{22}$

$$
\begin{aligned}
& \sigma=\frac{\lambda \Delta q}{2(4+\lambda)}\left[1+\frac{3 \lambda \Delta q}{2(2+\lambda)}\right], \\
& b=1+\frac{4 \lambda \Delta q}{4+\lambda}\left[1+\frac{2 \lambda(3+\lambda) \Delta q}{(2+\lambda)(4+\lambda)}\right],
\end{aligned}
$$

$$
c=\frac{-3}{1+f_{1}(\lambda) \Delta q+f_{2}(\lambda) \Delta q^{2}+f_{4 / \lambda}(\lambda) \Delta q^{4 / \lambda}},
$$

where $f_{1}=4 \lambda /(4-\lambda), f_{2}=\lambda^{3}(3-\lambda) /(2-\lambda)$, and $f_{4 / \lambda}=-4 \lambda$ / $(4-\lambda)-4 \lambda /(2-\lambda)-4(3-\lambda) /(1-\lambda)$.

Notice that $\beta_{p}$ in Eqs. (21) and (25) includes also the rotational part in Eq. (2b), and this part depends on the profiles of the rotation frequency $\Omega$ and plasma density $\rho_{0}$. In the comparison with numerical results in the next section we will use the following parabolic profiles of $\Omega(r)$ and $\rho_{0}(r):^{4}$

$$
\begin{aligned}
& \Omega(r)=\Omega(0)\left(1-\frac{r^{2}}{r_{\Omega}^{2}}\right), \\
& \rho_{0}(r)=\rho_{0}(0)\left(1-\frac{r^{2}}{r_{\rho}^{2}}\right) .
\end{aligned}
$$

We assume, in addition, that $r_{1} / r_{\Omega} \ll 1$ and $r_{1} / r_{\rho} \ll 1$ and retain only leading-order terms in $r / r_{\Omega}$ and $r / r_{\rho}$. After some simple algebra we obtain from Eq. (2b) $\beta_{p}=\beta_{p 0}+\beta_{p \Omega}$ where $\beta_{p 0}$ denotes the usual, static part of $\beta_{p}$ and

$$
\beta_{p \Omega}=\frac{\sigma_{2} \hat{\Omega}^{2}}{2}\left(\frac{2}{\varepsilon_{\Omega}^{2}}+\frac{1}{\varepsilon_{\rho}^{2}}\right) .
$$

Here $\varepsilon_{\rho}^{2}=\left(r_{\rho} / R_{0}\right)^{2}, \varepsilon_{\Omega}^{2}=\left(r_{\Omega} / R_{0}\right)^{2}$, and it is assumed that $\varepsilon_{\rho} \sim \varepsilon_{\Omega} \sim \varepsilon_{a}$ (or possibly larger, for instance $\varepsilon_{\rho}=\varepsilon_{\Omega}=\infty$ in the case of flat profiles).

Since an increasing $\beta_{p}$ in Eqs. (21) and (25) usually represents a destabilizing effect on the internal kink, ${ }^{11,21,22}$ we see that a decreasing rotation frequency and plasma density with minor radius both lead to a destabilizing effect through the enhanced, total beta. It will be seen in the next section that this effect becomes visible, both analytically and numerically, when $\delta \hat{W}_{\text {Bussac }}$ is large compared with $\delta \hat{W}_{\text {Rot1 }}$, i.e., for a sufficiently large $\Delta q$. When $\Delta q$ is small, on the other hand, this effect is overshadowed by the stronger profile effects included in $\delta \hat{W}_{\text {Rot } 1}$.

\section{B. Expression for $\delta \hat{W}_{\text {Rot1 }}$}

We now calculate the contribution to $\delta \hat{W}$ from the term involving $d A_{2} / d r$ in Eq. (15). Considering first the consistent case $\sigma_{1}=\sigma_{2}=1$, the coefficient $A_{2}$ is given by ${ }^{5,15}$

$$
\begin{aligned}
A_{2}= & -\frac{\omega_{D}^{2}}{\omega_{A}^{2}} \\
& -\frac{\left(3 \omega_{D}^{2}-2 \omega_{D} \Omega+\Omega^{2} / 2\right) \Omega^{2}+\left(2 \omega_{D}^{2}-4 \omega_{D} \Omega+\Omega^{2}\right) \omega_{s}^{2}}{\omega_{A}^{2}\left(\omega_{s}^{2}-\omega_{D}^{2}\right)} .
\end{aligned}
$$

The complete expression for $d A_{2} / d r$ becomes rather complex because of the $r$-dependence of all quantities appearing in Eq. (29). Since $\omega_{D} \sim \varepsilon^{2} \omega_{A} \sim \varepsilon \omega_{s}$, however, we can simplify $d A_{2} / d r$ by setting $\omega_{D}=0$ after the differentiation of $A_{2}$. Here it is important to include the contribution from $d \omega_{D} / d r=d \Omega / d r$ in the second, linear term in $\omega_{D}$ before we set $\omega_{D}=0$. This gives 


$$
\frac{d A_{2}}{d r} \cong-\frac{d}{d r}\left[\frac{\Omega^{2}}{\omega_{A}^{2}}\left(1+\frac{\mathcal{M}^{2}}{\Gamma}\right)\right]+\frac{2\left(\Omega^{2}\right)^{\prime}}{\omega_{A}^{2}}\left(1+\frac{\mathcal{M}^{2}}{\Gamma}\right) .
$$

We see that it is mainly the profiles of $\Omega(r)$ and $\omega_{A}^{2}(r)$ $=B_{0}^{2} /\left(\mu_{0} \rho_{0}(r) R_{0}^{2}\right)$, and to some extent also $\mathcal{M}^{2}(r)$, that determine $\delta \hat{W}_{\text {Rotl }}$. As will be seen in the examples in the next section, however, $\mathcal{M}^{2} / \Gamma \ll 1$ in most cases of interest, implying that these terms can be neglected in Eq. (30). We then get $d A_{2} / d r \cong-\mu_{0} R_{0}^{2} \Omega^{2} \rho_{0}^{\prime} / B_{0}^{2}+\left(\Omega^{2}\right)^{\prime} / \omega_{A}^{2}$ and

$$
\delta \hat{W}_{\mathrm{Rot} 1}=\frac{\mu_{0} R_{0}^{4}}{r_{1}^{4} B_{0}^{2}} \int_{0}^{r_{1}} r^{\prime 2}\left(\Omega^{2} \frac{d \rho_{0}}{d r^{\prime}}-\rho_{0} \frac{d \Omega^{2}}{d r^{\prime}}\right) d r^{\prime} .
$$

Hence, a decreasing density with minor radius inside $r=r_{1}$ is seen to lead to a negative and destabilizing contribution to $\delta \hat{W}_{\text {Rot1 }}$ whereas a decreasing rotation frequency with $r$ leads to a positive and therefore stabilizing contribution to $\delta \hat{W}_{\text {Rot1 }}$ (and vice versa for profiles that are increasing with the minor radius). For the profiles in Eqs. (27a) and (27b) we get in particular

$$
\delta \hat{W}_{\mathrm{Rot} 1}=\frac{\hat{\Omega}^{2}}{2}\left(\frac{2}{\varepsilon_{\Omega}^{2}}-\frac{1}{\varepsilon_{\rho}^{2}}\right) .
$$

This result is identical to the contribution $W_{\text {Erot }}+W_{\text {flutter }}$ to the internal energy calculated by Waelbroeck, ${ }^{4}$ and apparently the same effect is included in the coefficient $A_{2}$ in Eq. (29). This contribution from $A_{2}$ was not explicitly calculated in Ref. 5 , but it was calculated in a later paper, ${ }^{23}$ dealing with the isothermal case $\Gamma=1$. There, however, the second term in Eq. (30) was omitted, and therefore the final expression for $\delta W$ obtained in Ref. 23 is incomplete, and the conclusions drawn on the basis of this, incomplete form of $\delta W$ incorrect. Similarly, the analysis of Mercier modes and the Mercier criterion derived for an isothermal plasma, satisfying $\Gamma=1$, in Ref. 24 is incomplete in the same respect. With the exception of the small part of that paper dealing with the case $\Gamma=1$, this does not affect the rest of the analysis and conclusions in Ref. 24.

Let us now consider the inconsistent case $\sigma_{1}=\sigma_{2}=0$. First we notice that there is no dependence on $q$ in the expressions for $A_{2}$ and $d A_{2} / d r$ in Eqs. (29) and (30). From the (very long) corresponding expression for $d A_{2} / d r$ obtained with help of computer algebra in the inconsistent case, it turns out that this is no longer valid. To simplify this term in the inconsistent case we therefore assume that $\Delta q$ is small and set $q=1$. Using this approximation, and setting also $\sigma_{1}=0$ in the remaining expression for $d A_{2} / d r$ (which is independent of $\sigma_{2}$ ), it turns out that $d A_{2} / d r$ becomes very similar to the expression in Eq. (30). More precisely, the terms of order $\Omega^{2}$ are the same as in Eq. (30) whereas the terms of order $\Omega^{4}$ are somewhat different. Since these terms anyway are neglected here, it follows that the leading-order rotational contribution to $\delta \hat{W}$ from the $d A_{2} / d r$ term is given by the expressions for $\delta \hat{W}_{\text {Rot1 }}$ in Eqs. (31) and (32) also in the inconsistent case $\sigma_{1}=\sigma_{2}=0$.

\section{Expression for $\delta \hat{W}_{\text {Rot2 }}$}

Finally, we calculate the contribution to $\delta \hat{W}$ from the terms involving the factor $\sigma_{2}-1$ in Eqs. (4) and (9). With the parabolic profiles of $\Omega$ and $\rho_{0}$ given by Eqs. (27a) and (27b), and again retaining only leading-order terms in $r / r_{\Omega}$ and $r / r_{\rho}$, we see that Eq. (4) can be written in the form

$$
\mathcal{L}_{2} \xi_{2}+\frac{d}{d r}\left(r^{3} T_{1} \frac{d \xi_{1}}{d r}\right)+r^{2} \widetilde{T}_{2} \frac{d \xi_{1}}{d r}=0
$$

where

$$
\begin{aligned}
& \widetilde{T}_{2}=\left(6 \mu^{2}-6 \mu+3\right) \Delta^{\prime}+\left(2 \mu^{2}-4 \mu+\frac{7}{2}+G\right) \frac{r}{R_{0}}, \\
& G=\left(\sigma_{2}-1\right) \hat{\Omega}^{2}\left(\frac{4}{\varepsilon_{\Omega}^{2}}+\frac{2}{\varepsilon_{\rho}^{2}}\right) .
\end{aligned}
$$

Following the analysis in Ref. 5 we consider the jump conditions for $\xi_{2}$ and $\xi_{2}^{\prime}$ at $r=r_{1}$. For the top hat eigenfunction $\xi_{1}=$ const $=\hat{\xi}$, explained in connection with Eq. (15), we get from Eq. (33),

$$
\begin{aligned}
& {\left[\xi_{2}\right]=T_{1}\left(r_{1}\right) \hat{\xi}=\left(-\Delta^{\prime}+\frac{r_{1}}{2 R_{0}}\right) \hat{\xi},} \\
& {\left[\xi_{2}^{\prime}\right]=\widetilde{T}_{2}\left(r_{1}\right) \frac{\hat{\xi}}{r_{1}}=\left[3 \Delta^{\prime}+\left(\frac{3}{2}+G\right) \frac{r_{1}}{R_{0}}\right] \hat{\xi} .}
\end{aligned}
$$

It follows from Eq. (33) that $\xi_{2}$ fulfills the homogeneous equation $\mathcal{L}_{2} \xi_{2}=0$ outside $r=r_{1}$, and we can write $\xi_{2}=c_{2}^{ \pm} x_{2}^{ \pm}$ where $x_{2}^{ \pm}$are normalized solutions to $\mathcal{L}_{2} x_{2}^{ \pm}=0$ for $r \geq r_{1}\left(x_{2}^{+}\right)$ and $r \leq r_{1}\left(x_{2}^{-}\right)$satisfying $x_{2}^{ \pm}\left(r_{1}\right)=1$. From the jump conditions above, and writing $\Delta^{\prime}=-\left(r / R_{0}\right) \Lambda$, where $\Lambda=\beta_{p}+\ell_{i} / 2$, we then get

$$
c_{2}^{-}=\hat{\xi} \frac{r_{1}}{R_{0}} \frac{c(1 / 2+\Lambda)-(3 / 2-3 \Lambda+G)}{b-c},
$$

where $b$ and $c$ are given by Eq. (23). The quantity $c_{2}^{+}$is given by a similar expression but is not needed here. Using Eq. (37) and the fact that $\xi_{2}=c_{2}^{-} x_{2}^{-}$in the region $r \leq r_{1}$ we can now calculate all terms on the right hand side of Eq. (15) involving $\sigma_{2}-1$. Looking first at the terms involving $W_{1}-W_{5}$, we get by using Eqs. (8) and (23),

$$
\begin{aligned}
-\hat{\xi} \int_{0}^{r_{1}} W_{1} d r-\left.r_{1}^{3} W_{2} \frac{d \xi_{1}}{d r}\right|_{r=r_{1}}-\left.r_{1}^{3} W_{4} \frac{d \xi_{2}}{d r}\right|_{r=r_{1}}-\left.r_{1}^{2} W_{5} \xi_{2}\right|_{r=r_{1}} \\
=-\frac{r_{1}^{4} \hat{\xi}}{4 R_{0}^{2}}\left(3 \beta_{p}+\frac{1}{2} \ell_{i}-\frac{1}{4}\right) \\
-\frac{r_{1}^{4}}{R_{0}}\left(\frac{1}{4} \Lambda+\frac{1}{8}\right) \frac{c_{2}^{-} b}{r_{1}}-\frac{r_{1}^{3}}{R_{0}}\left(\frac{3}{4} \Lambda-\frac{3}{8}\right) c_{2}^{-} .
\end{aligned}
$$

This expression includes both $\delta \hat{W}_{\text {Bussac }}$ in Eq. (21) plus a

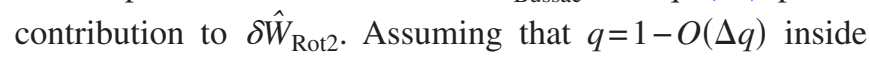
$r=r_{1}$ and neglecting terms of order $\Delta q$, the other terms in Eq. (15) involving $\sigma_{2}-1$ become 


$$
\left.\begin{array}{l}
\frac{\left(\sigma_{2}-1\right) \mu_{0} R_{0}^{2}}{4 B_{0}^{2}} \hat{\xi} \int_{0}^{r_{1}} r^{2}(2 / q-5)\left(\rho_{0} \Omega^{2}\right)^{\prime} d r \\
=\frac{3 G r_{1}^{4} \hat{\xi}}{16 R_{0}^{2}}+O(\Delta q), \\
-\frac{\left(\sigma_{2}-1\right) \mu_{0} R_{0}^{3}}{B_{0}^{2}}\left\{\frac{1}{2}\left[r^{2}\left(\rho_{0} \Omega^{2}\right)^{\prime} \xi_{2}\right]_{r=r_{1}}\right. \\
-\frac{1}{8}\left[r^{2}\left(\rho_{0} \Omega^{2}\right)^{\prime}\left((4 / q-2) \Delta^{\prime}-\frac{r}{R_{0}}\right) \xi_{1}\right]_{r=r_{1}}
\end{array}\right\}
$$

Since $\Delta q=1-q_{0}$ is assumed to be small here, we use small- $\Delta q$ expansions of $b, c$, and $\ell_{i}$ in $\Lambda$ and $c_{2}^{-}$also in Eqs. (38) and (39). Assuming also $\lambda=2$ we get from Eq. (26) $b=1+4 \Delta q / 3, c=-3+12 \Delta q$, and in addition $\ell_{i}=1 / 2+\Delta q / 3$. By using these expressions and adding the terms in Eqs. (38) and (39) we find, after removing the part belonging to $\delta \hat{W}_{\text {Bussac }}$, that $\delta \hat{W}_{\text {Rot } 2}=\beta_{p} G / 2-G^{2} / 8+O(\Delta q G)$. However, as will be seen in the next section, $\hat{\Omega}=\Omega(0) / \omega_{A}(0)$ is always much smaller than $\varepsilon$ in the relevant regime of $\hat{\Omega}$. For such rotation frequencies, $G$ in Eq. (35) is a sufficiently small quantity that the term $-G^{2} / 8$ can be neglected. The final expression for $\delta \hat{W}_{\text {Rot2 }}$ then becomes

$$
\delta \hat{W}_{\text {Rot } 2}=\left(\sigma_{2}-1\right) \beta_{p} \hat{\Omega}^{2}\left(\frac{2}{\varepsilon_{\Omega}^{2}}+\frac{1}{\varepsilon_{\rho}^{2}}\right) .
$$

We point out that since $\beta_{p}$ is never particularly large, the omitted term of order $\Delta q G$ in $\delta \hat{W}_{\text {Rot2 }}$ easily becomes of similar magnitude as $\delta \hat{W}_{\mathrm{Rot} 2}=\beta_{p} G / 2$ in Eq. (40), unless $\Delta q$ indeed is very small $\left(\Delta q \ll \beta_{p}\right)$. In the comparison with numerical results in the inconsistent case $\sigma_{1}=\sigma_{2}=0$ it is therefore necessary to use a sufficiently small $\Delta q$ in order to get a good agreement. Furthermore, $\beta_{p}$ in Eq. (40) includes, in principle, also the rotational enhancement in Eq. (28). However, since $\delta \hat{W}_{\text {Rot2 }}$ is of interest essentially only when $\sigma_{2}=0, \beta_{p}$ in Eq. (40) in practice stands for the static part $\beta_{p 0}$.

It is seen that, by not including the rotation-enhanced Shafranov shift in Eq. (2b), i.e., using $\sigma_{2}=0, \delta \hat{W}_{\text {Rot2 }}$ in Eq. (40) becomes negative and destabilizing. On the other hand, it is easily seen from the analysis above that the quantity $2 / \varepsilon_{\Omega}^{2}+1 / \varepsilon_{\rho}^{2}$ in Eq. (40) actually comes from the gradient of $\rho_{0} \Omega^{2}=\rho_{0}(0) \Omega^{2}(0)\left[1-\left(2 / r_{\Omega}^{2}+1 / r_{\rho}^{2}\right) r^{2}+\ldots\right]$ and for (usual) profiles such that $\left(\rho_{0} \Omega^{2}\right)^{\prime}<0$ we have $2 / \varepsilon_{\Omega}^{2}+1 / \varepsilon_{\rho}^{2}>0$, leading to a destabilizing effect from $\delta \hat{W}_{\text {Rot2 }}$. For (unusual) profiles satisfying $\left(\rho_{0} \Omega^{2}\right)^{\prime}>0$, on the other hand, the quantity $2 / \varepsilon_{\Omega}^{2}+1 / \varepsilon_{\rho}^{2}$ instead becomes negative, leading to a stabilizing effect from not including the rotation-dependent part of Shafranov shift in the analysis.

Before we compare the analytical growth rate in Eq. (20) with numerically computed growth rates using consistent as well as inconsistent equilibria in the next section, we discuss the relative magnitudes of the three different parts of $\delta \hat{W}$ in Eq. (16). First, it is clear that $\delta \hat{W}_{\text {Rot1 }}$ and $\delta \hat{W}_{\text {Rot2 }}$ are of the same order of magnitude, provided that $\beta_{p} \sim 1$. Furthermore, if $\delta \hat{W}_{\text {Bussac }} \sim 1$, rotation velocities of the order of $\hat{\Omega} \sim \varepsilon$ would be required in order for $\delta \hat{W}_{\text {Rot1,2 }}$ to be of the same order as $\delta \hat{W}_{\text {Bussac. Supposing, in addition, that all quantities }}$ appearing in Eq. (20), except for $\varepsilon_{1}$ and $\hat{\Omega}$, are of order unity it is seen that rotational stabilization due to the second term (second GAM frequency) indeed would occur for $\hat{\Omega} \sim \varepsilon$ (if $\left.\sigma_{1}>0\right)$. It should be noticed, however, that the GAM frequency also involves the quantity $\beta_{0}$ in the denominator, and that the present analysis is based on the ordering $\beta_{0} \sim \varepsilon^{2}$. For very small $\varepsilon$, the GAM stabilization will therefore occur at the lower rotation frequency $\hat{\Omega} \sim \varepsilon \beta_{0}^{1 / 4} \sim \varepsilon^{3 / 2}$. At such rotation frequencies we see that $\delta \hat{W}_{\text {Rot1,2 }}$ both become a factor $\varepsilon$ smaller than $\delta \hat{W}_{\text {Bussac }}$. For sufficiently small $\varepsilon$ the effects of $\delta \hat{W}_{\text {Rot } 1,2}$ should therefore be negligible and a stability analysis based on $\delta \hat{W}_{\mathrm{Bussac}}$ and $\omega_{\mathrm{GAM} 2}^{2}=\omega_{\mathrm{BV}}^{2}$ is sufficient. This is what is seen in Fig. 1, where $\varepsilon_{a}=0.1$. For realistic values of $\varepsilon_{a}$, however, and especially in equilibria where $\Delta q$ is not too large so that $\delta \hat{W}_{\text {Bussac }} \sim \Delta q$ is also a small quantity, it turns out that the profile effects included in $\delta \hat{W}_{\text {Rot1 }}$ (which is also finite in the consistent case) are very important and have to be taken into account in spite of the fact that they are formally of higher order in $\varepsilon$.

\section{COMPARISON OF ANALYTICAL AND NUMERICAL GROWTH RATES}

For a given shape of the pressure profile, the quantities $\beta_{0}$ and $\beta_{p}$ appearing in Eq. (20) are not independent. In the comparison studies in this section, the parabolic pressure profile $p_{0}(r) / p_{0}(0)=1-r^{2} / a^{2}$ has been used. For this pressure profile it follows from Eq. (2b) that $\beta_{0}=2 \beta_{p 0} \varepsilon_{a}^{2}$, leading to $\beta_{0}=0.6 \%$ for the combination $\varepsilon_{a}=0.1$ and $\beta_{p 0}=0.3$ used in Fig. 1 in Sec. I and in Figs. 3 and 4 in this section. For the combination $\varepsilon_{a}=0.3$ and $\beta_{p 0}=0.3$, used in several other figures in this section, we get instead $\beta_{0}=5.4 \%$.

We first look at the analytical growth rate in the inconsistent case $\sigma_{1}=\sigma_{2}=0$ and compare with numerical results obtained from MISHKA-F, with (static) equilibria provided by the HELENA code. ${ }^{25}$ When $\sigma_{1}=0$ the stabilizing effect from the GAM frequency in Eq. (20) vanishes, and rotational effects appear only in $\delta \hat{W}_{\text {Rot1 }}$ and $\delta \hat{W}_{\text {Rot2 }}$. The combined effect from these two terms becomes

$$
\delta \hat{W}_{\text {Rot } 1}+\delta \hat{W}_{\text {Rot } 2}=\hat{\Omega}^{2}\left(\frac{1-2 \beta_{p}}{\varepsilon_{\Omega}^{2}}-\frac{0.5+\beta_{p}}{\varepsilon_{\rho}^{2}}\right),
$$

where $\beta_{p}$ includes the static part only, since the rotational contribution to $\beta_{p}$ in Eq. (28) also vanishes. For certain combinations of $\beta_{p}$ and the profile parameters $\varepsilon_{\Omega}^{2}$ and $\varepsilon_{\rho}^{2}$, the total effect from the rotation in Eq. (41) apparently vanishes. This occurs for instance when the density profile is flat $\left(\varepsilon_{\rho}^{2}=\infty\right)$ and $\beta_{p}=0.5$, independently of $\varepsilon_{\Omega}^{2}$. Furthermore, for $\varepsilon_{\rho}^{2}=\infty$ and $\varepsilon_{\Omega}^{2}>0$ (i.e., a rotation frequency decreasing with minor radius), the flow is stabilizing for $\beta_{p}<0.5$ and destabilizing 


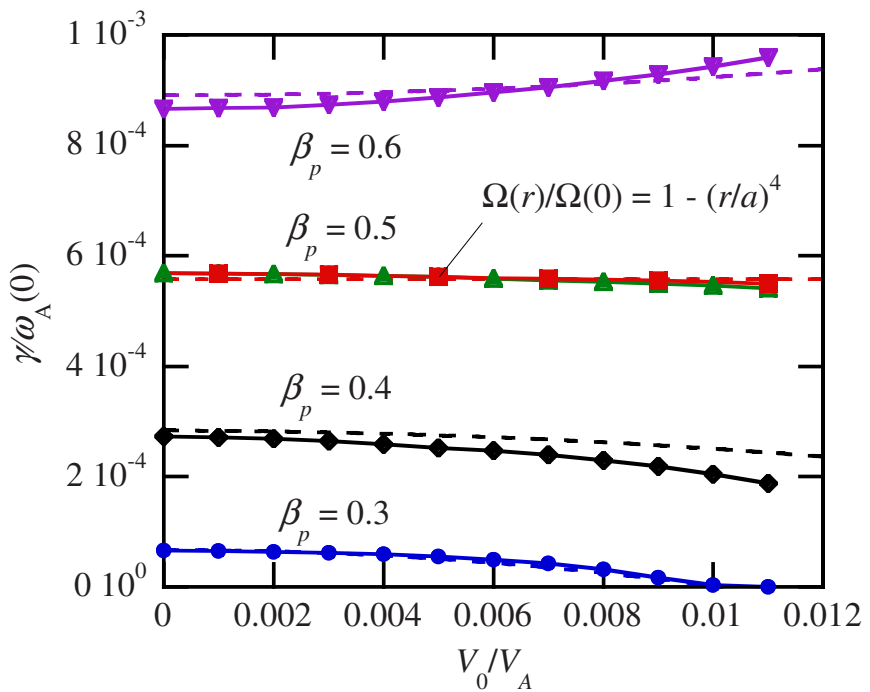

FIG. 2. (Color online) Growth rate vs rotation velocity at the axis for an inconsistent equilibrium $\sigma_{1}=\sigma_{2}=0$ with $\varepsilon_{a}=0.1, q_{0}=0.95, r_{1} / a=0.3$, and different $\beta_{p}$. The density is flat and the rotation profile parabolic. In addition, for $\beta_{p}=0.5$ the growth rate is computed also for the rotation profile $\Omega(r) / \Omega(0)=1-(r / a)^{4}$, shown by the squares. The dashed curves are calculated analytically and the symbols with MISHKA-F.

for $\beta_{p}>0.5$. This effect is seen in Fig. 2, where analytical growth rates from Eq. (20) for different $\beta_{p}$ are shown as functions of the flow velocity at the axis $\left(V_{0} / V_{A} \equiv \hat{\Omega}\right)$ by the dashed curves, and the corresponding growth rates calculated with MISHKA-F are shown by the different symbols. The rotation profile is given by $\varepsilon_{\Omega}=\varepsilon_{a}=0.1$, and for $\beta_{p}=0.5$ the numerical growth rate is shown also for the profile $\Omega(r) / \Omega(0)=1-r^{4} / a^{4}$, by the squares. Other parameters in this plot are $\Delta q=0.05$ and $r_{1} / a=0.3$, and the static (Bussac) part of the analytical growth rate has been calculated from Eq. (25) (without any correction factor in this case).

Other combinations of $\varepsilon_{\Omega}^{2}, \varepsilon_{\rho}^{2}$, and $\beta_{p}$ that nullify the rotational contribution in Eq. (41) are, for instance, flat profiles of both $\Omega$ and $\rho_{0}\left(\varepsilon_{\Omega}^{2}=\varepsilon_{\rho}^{2}=\infty\right)$ and profile parameters satisfying $\varepsilon_{\Omega}^{2} / \varepsilon_{\rho}^{2}=\left(1-2 \beta_{p}\right) /\left(0.5+\beta_{p}\right)$. Taking, for instance, $\beta_{p}=0.3$, the rotational effect should vanish for $\varepsilon_{\Omega}=\varepsilon_{\rho} / \sqrt{2}$. In Fig. 3 we illustrate both the case with flat profiles of both $\rho_{0}$ and $\Omega$ and a case where the profiles are given by $\rho_{0}(r) / \rho_{0}(0)=1-r^{2} / a^{2}$ and $\Omega(r) / \Omega(0)=1-2 r^{2} / a^{2}$. Other parameters in this plot are $\varepsilon_{a}=0.1, \Delta q=0.062$, and $r_{1} / a=0.3$, and the static part of the analytical growth rate is again calculated from Eq. (25). The numerical growth rates in the inconsistent case $\sigma_{1}=\sigma_{2}=0$ are seen to be essentially independent of the rotation velocity in both cases, as predicted by the analytical theory. For comparison, the growth rate using a self-consistent equilibrium, $\sigma_{1}=\sigma_{2}=1$, is shown by the dashed curves for both of the cases in the same figure, and, in addition, the numerical growth rate in the case of flat profiles of both $\rho$ and $\Omega$ are shown by the symbols. These numerical results are obtained with CASTOR-FLOW, which uses flowing equilibria from the DIVA code. ${ }^{26}$ Furthermore, $\beta_{0}=0.66 \%$ is used in the consistent case and other parameters are the same as for the curves calculated with $\sigma_{1}=\sigma_{2}=0$ in the same figure.

It is clear that the improvement in stability from the

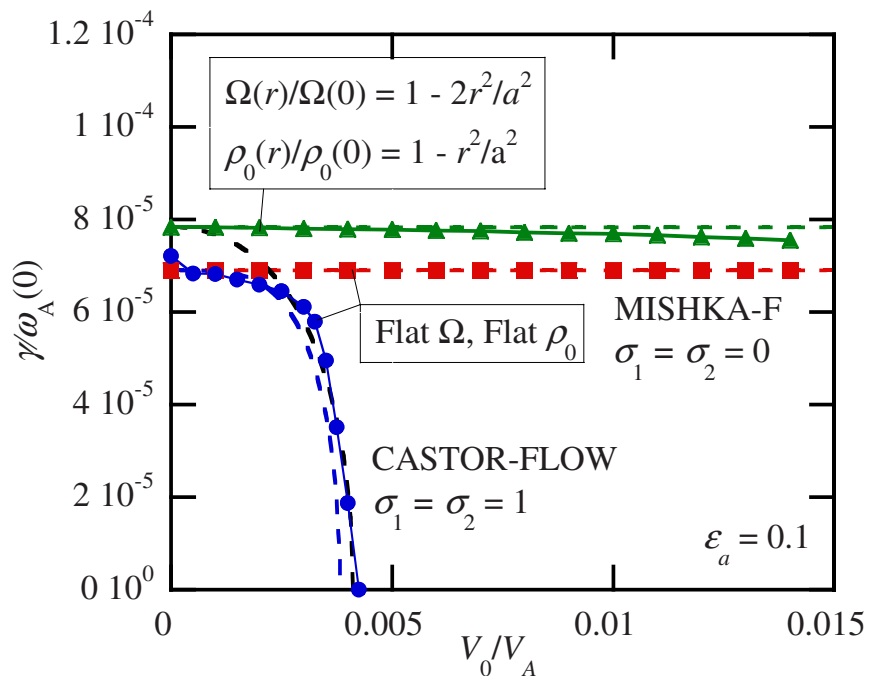

FIG. 3. (Color online) Growth rate vs rotation velocity at the axis for two combinations of the profiles of $\rho_{0}$ and $\Omega$ such that the flow does not modify the static growth rate when an inconsistent equilibrium $\left(\sigma_{1}=\sigma_{2}=0\right)$ is used. For comparison, the corresponding analytical growth rates in the consistent case $\left(\sigma_{1}=\sigma_{2}=1\right)$ are also shown for these two combinations of profiles, together with the CASTOR-FLOW result for the case when the profiles of both $\rho_{0}$ and $\Omega$ are flat. The plasma parameters are $\varepsilon_{a}=0.1, q_{0}=0.938, r_{1} / a=0.3$, $\beta_{p 0}=0.3$, and $\beta_{0}=0.66 \%$. The dashed curves are calculated analytically and the symbols with the numerical codes indicated.

inconsistent case in Fig. 1 to the inconsistent case with $\rho_{0}(r) / \rho_{0}(0)=1-r^{2} / a^{2}$ and $\Omega(r) / \Omega(0)=1-2 r^{2} / a^{2}$ in Fig. 3 is due to stronger rotation shear, or smaller $\varepsilon_{\Omega}$. Further improvement of the stability in the inconsistent case can be achieved by making the density profile more flat, but keeping a parabolic rotation profile. Figure 4 shows analytical and numerical growth rates for $\sigma_{1}=\sigma_{2}=0$ as well as for $\sigma_{1}=\sigma_{2}$

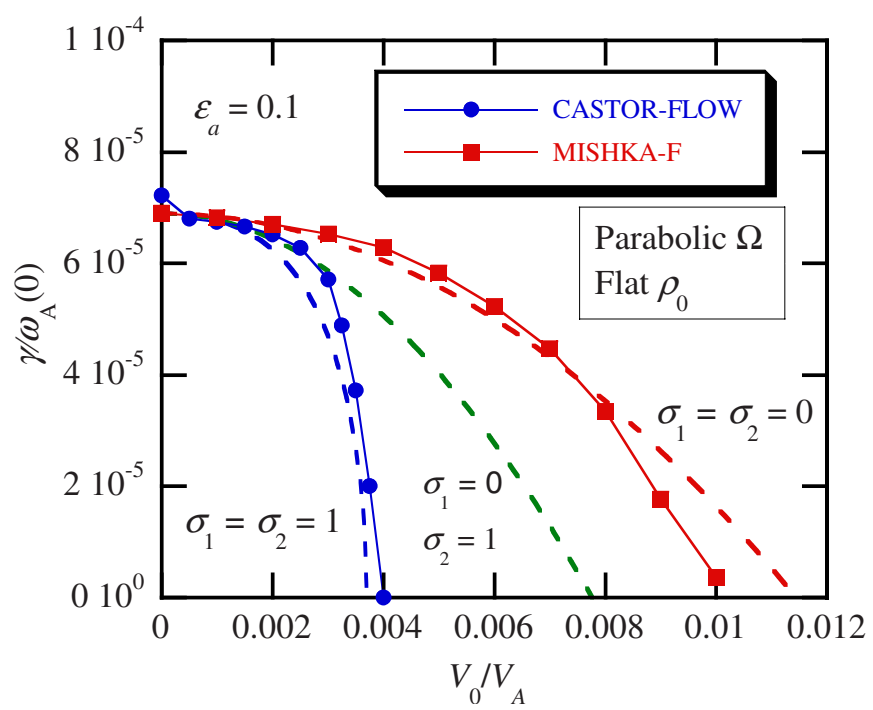

FIG. 4. (Color online) Growth rate vs rotation velocity at the axis in the case of a flat density and a parabolic rotation profile with $\varepsilon_{\Omega}=\varepsilon_{a}=0.1$. Other plasma parameters are $q_{0}=0.938, r_{1} / a=0.3, \beta_{p 0}=0.3$, and $\beta_{0}=0.66 \%$. Here, stabilization occurs both for inconsistent $\left(\sigma_{1}=\sigma_{2}=0\right)$ and consistent $\left(\sigma_{1}=\sigma_{2}=1\right)$ equilibria. In the intermediate case $\sigma_{1}=0$ and $\sigma_{2}=1$ the stabilizing GAM frequency is neglected but the stabilizing effect from the enhanced Shafranov shift in Eq. (2b) is included. The dashed curves are calculated analytically and the symbols with the numerical codes indicated. 


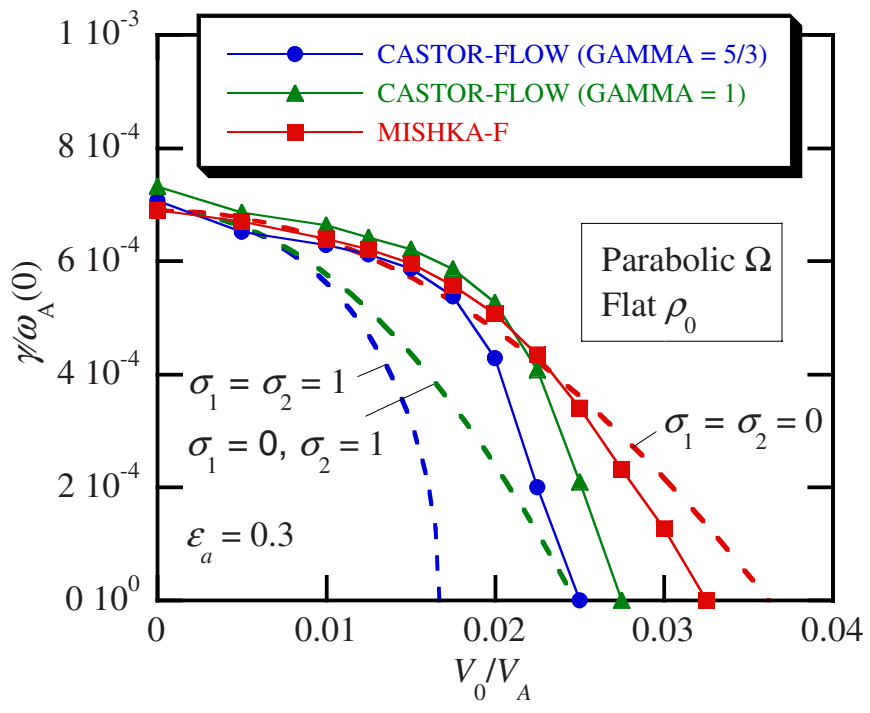

FIG. 5. (Color online) Growth rate vs rotation velocity at the axis in the case of a flat density and a parabolic rotation profile with $\varepsilon_{\Omega}=\varepsilon_{a}=0.3$. Other plasma parameters are $q_{0}=0.938, r_{1} / a=0.3, \beta_{p 0}=0.3$, and $\beta_{0}=5.6 \%$ and stabilization occurs both for inconsistent $\left(\sigma_{1}=\sigma_{2}=0\right)$ and consistent $\left(\sigma_{1}=\sigma_{2}=1\right)$ equilibria. The intermediate case $\sigma_{1}=0$ and $\sigma_{2}=1$ is simulated numerically by running CASTOR-FLOW with the adiabatic constant $\Gamma=1$, and the result is shown by the triangles. The dashed curves are calculated analytically and the symbols with the numerical codes indicated.

$=1$, for a flat density profile and the parabolic rotation profile $\Omega(r) / \Omega(0)=1-r^{2} / a^{2}$. Other parameters are the same as in the previous figure, i.e., $\varepsilon_{a}=0.1, \Delta q=0.062, r_{1} / a=0.3$, $\beta_{p 0}=0.3$, and $\beta_{0}=0.66 \%$. The combination of a flat density and a parabolic rotation profile (and not too large $\beta_{p}$, see Fig. 2) thus leads to rotational stabilization also in the inconsistent case, but the additional stabilization from the GAM frequency in the consistent case is seen to be significant. However, for these profiles a substantial part of the stabilization in the consistent case also comes from the enhanced Shafranov shift, as can be seen from the analytical curve with $\sigma_{1}=0$ and $\sigma_{2}=1$, a combination that removes both the stabilizing effect from the GAM frequency and the destabilizing effect from $\delta \hat{W}_{\text {Rot2 }}$.

Since the effect of using $\sigma_{1}=0$ and $\sigma_{2}=1$ mainly is to eliminate the stabilizing continuum (GAM) frequency in Eq. (13b), while the effect from the enhanced Shafranov shift in Eq. (2b) is still present, this case can be simulated numerically by running CASTOR-FLOW with an adiabatic constant $\Gamma=1$. Such a case is shown in Fig. 5, which is a similar plot as in Fig. 4 but with a larger $\varepsilon_{a}=0.3$ and with $\beta_{0}=5.4 \%$. The other parameters and profiles are the same as in Fig. 4. It is seen that the analytical curves are qualitatively similar to the numerical curves, but differ somewhat quantitatively due to the larger value of $\varepsilon_{a}$ here compared with Fig. 4. Figure 6 shows a comparison at the larger values of $\varepsilon_{a}=0.3$ and $\beta_{0}=5.4 \%$ also for the other two cases shown in Figs. 1 and 3, viz. the combinations of (i) parabolic flow and parabolic density $\left(\varepsilon_{\Omega}=\varepsilon_{\rho}=\varepsilon_{a}\right)$ and (ii) flat flow and flat density $\left(\varepsilon_{\Omega}=\varepsilon_{\rho}=\infty\right)$. Again, the analytical result in the consistent case agrees qualitatively with the numerical result but the numerical, stabilizing rotation frequency is approximately a factor 1.5 larger than the analytical prediction.

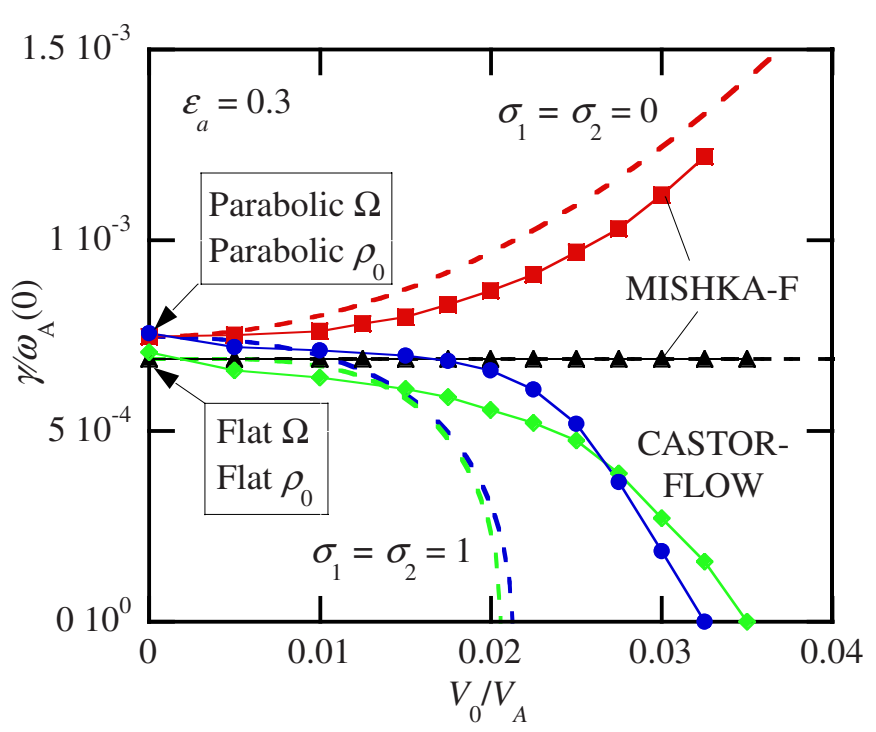

FIG. 6. (Color online) Growth rate vs rotation velocity at the axis for two combinations of the profiles of $\rho_{0}$ and $\Omega$ in a plasma with $\varepsilon_{a}=0.3$, $q_{0}=0.938, r_{1} / a=0.3, \beta_{p 0}=0.3$, and $\beta_{0}=5.6 \%$. Results both for inconsistent (upper curves) and consistent (lower curves) equilibria are shown. The dashed curves are calculated analytically and the symbols with the numerical codes indicated.

As an illustration of the discussion at the end of Sec. II on the relative magnitudes of the different parts in $\delta \hat{W}$ in Eq. (16) and the dependence of the profile effects described by $\delta \hat{W}_{\text {Rot1 }}$ on the aspect ratio, we plot the (consistent) critical rotation velocity required for stabilization as a function of $\varepsilon_{a}$ from Eq. (20) in Fig. 7. All four combinations of parabolic and flat density and rotation profiles are included in this figure and we use the same parameters as before, i.e., $\Delta q=0.062, r_{1} / a=0.3, \beta_{p 0}=0.3$, and $\beta_{0}=2 \beta_{p 0} \varepsilon_{a}^{2}$. It is seen

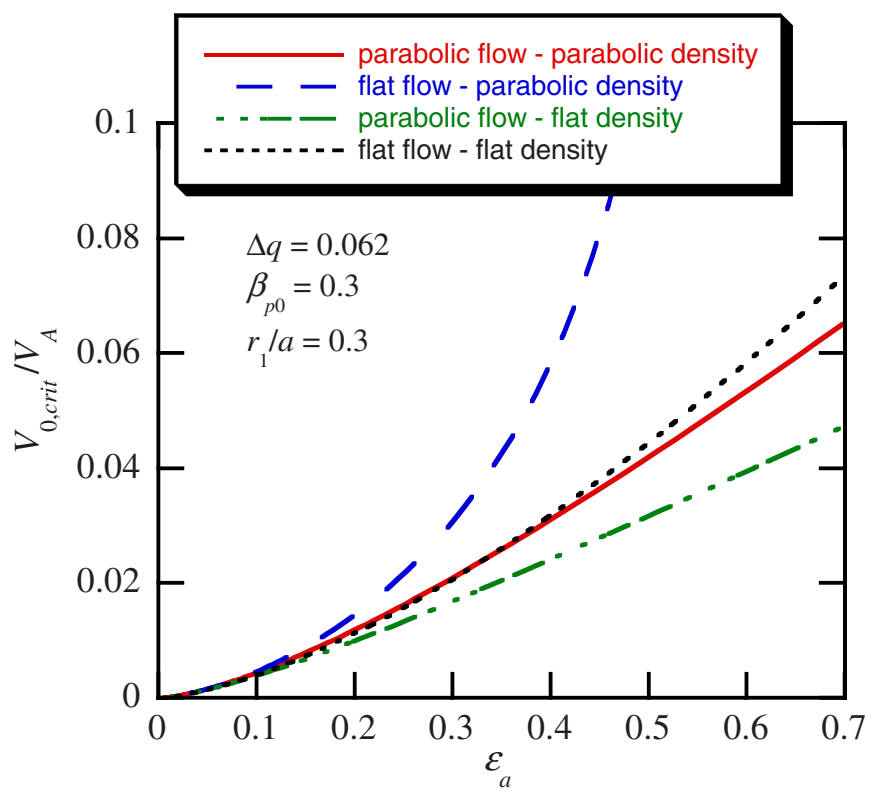

FIG. 7. (Color online) Critical rotation velocity $V_{0 \text {,crit }}$ required for stabilization of the kink mode vs the inverse aspect ratio $\varepsilon_{a}$. $V_{0 \text {,crit }}$ is shown for the four combinations of the profiles of $\rho_{0}$ and $\Omega$ indicated and is calculated from Eq. (20) using $\sigma_{1}=\sigma_{2}=1, q_{0}=0.938, r_{1} / a=0.3, \quad \beta_{p 0}=0.3$, and $\beta_{0}=2 \beta_{p 0} \varepsilon_{a}^{2}$. 


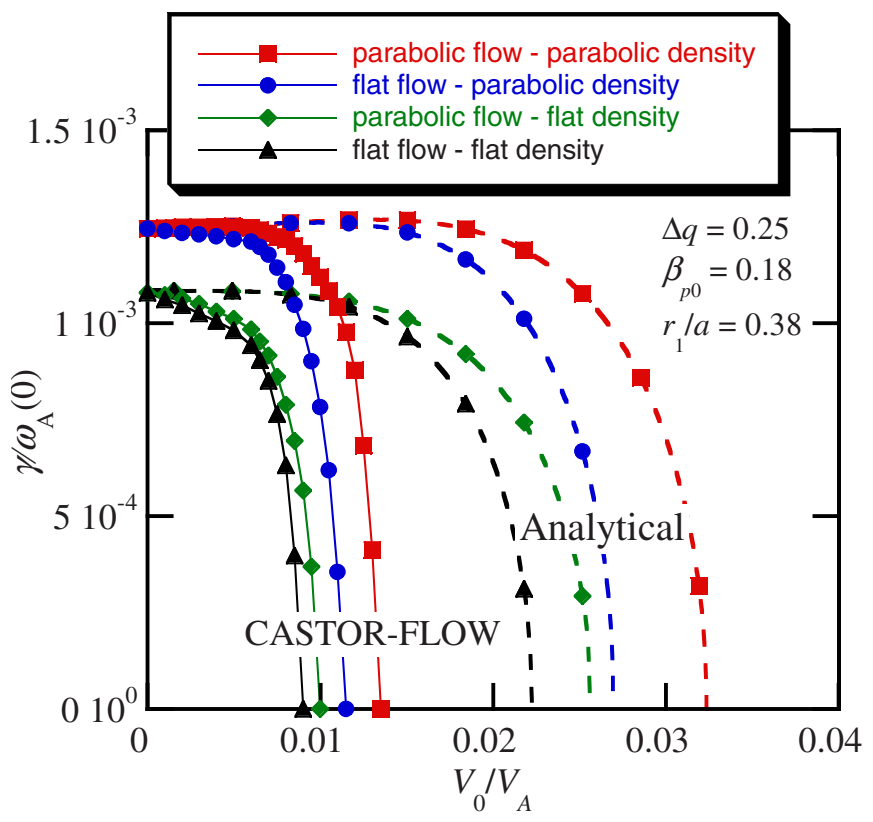

FIG. 8. (Color online) Growth rate vs rotation velocity at the axis for four different combinations of the profiles of $\rho_{0}$ and $\Omega$ in a plasma with $\varepsilon_{a}=0.3, q_{0}=0.75, r_{1} / a=0.38, \beta_{p 0}=0.3$, and $\beta_{0}=3.25 \%$. The flow is treated consistently and the solid lines show CASTOR-FLOW results whereas the dashed lines are predicted by the analytical theory.

that, as expected, the profile effects become less important with increasing aspect ratio, and that the scaling $\hat{\Omega}_{\text {crit }} \sim \varepsilon_{a}^{3 / 2}$ prevails as $\varepsilon_{a}$ becomes very small. In practice, however, the profile effects included in $\delta \hat{W}_{\text {Rot1 }}$ become significant already for $\varepsilon_{a}=0.1-0.2$ and very strong at realistic values of $\varepsilon_{a}{ }^{19}$ We emphasize that these analytical curves are valid only in a qualitative sense for $\varepsilon_{a}>0.2-0.3$, and that this qualitative dependence of the critical rotation on the profiles is valid only for equilibria with sufficiently small $\Delta q$.

With increasing $\Delta q$, the magnitude of $\delta \hat{W}_{\text {Bussac }}$ increases, leading to a smaller influence of $\delta \hat{W}_{\text {Rot1 }}$ and the profile effects described by this term. Instead, the profile effects on $\beta_{p}$ expressed by Eq. (28) become more important and since a parabolic flow profile leads to an increasing total $\beta_{p}$, and thereby a destabilizing effect through a larger $\left|\delta \hat{W}_{\text {Bussac }}\right|$, it turns out that for sufficiently large $\Delta q$ the total effect of a parabolic flow profile can become destabilizing in spite of its stabilizing effect in $\delta \hat{W}_{\text {Rot1 }}$. As to the total effect of the density profile, since a decreasing density with minor radius also increases $\beta_{p}$, this effect reinforces the destabilizing effect that such a density profile already has in $\delta \hat{W}_{\text {Rot1 }}$, according to Eqs. (31) and (32). These effects can be seen in Fig. 8, which shows the growth rates in the consistent case $\sigma_{1}=\sigma_{2}=1$ in a plasma with the parameters $\Delta q=0.25, r_{1} / a=0.38, \varepsilon_{a}=0.3$, $\beta_{p}=0.18$, and $\beta_{0}=3.25 \%$. All four combinations of parabolic and flat density and rotation profiles are included in the figure, with analytical results shown by the dashed curves and numerical (CASTOR-FLOW) results by the solid curves. By matching the shear at $r=r_{1}$ in the numerical $q$-profile $\left(s_{1}=0.83\right)$ to the analytical $q$-profile in Eq. (24), we get an equivalent $\lambda=3.32$. In the figure, the analytical growth rates are shown for $\lambda \approx 3.4$ in the expression for $\delta \hat{W}_{\text {Bussac }}$ in Eqs. (21) and (26). It is seen that the profile effects expressed by the enhanced $\beta_{p}$ in the analytical theory agree qualitatively with the numerical results. Quantitatively, however, the analytical theory predicts a critical rotation required for stabilization exceeding the numerical by a factor of the order of two. The figure nevertheless illustrates the fact that, when $\Delta q$ becomes large enough, the combination of parabolic flow and parabolic density is the most unstable one whereas the case when both profiles are flat is easiest to stabilize with the flow. Additional examples and details regarding profile effects are given in Ref. 19.

\section{NUMERICAL GROWTH RATES USING EXPERIMENTAL PROFILES}

We now look at the numerical growth rate of the internal $n=1$ mode as a function of rotation velocity in two realistic, experimental situations and compare the results obtained by using a plasma equilibrium without centrifugal effects included with the results obtained by taking such effects into account. Due to the more complex nature of the realistic equilibria in comparison with the analytical model in Sec. II, e.g., in terms of the profiles of density and rotation velocity, finite aspect ratio, and significant shaping effects, we do not attempt to model these growth rates analytically here. It will be seen that the results nevertheless are qualitatively similar to the analytical results obtained in the previous section.

\section{A. MAST analysis}

The stability of the $n=1$ internal kink mode has been assessed in a typical MAST H-mode plasma using both the inconsistent and consistent treatments of the equilibrium plasma rotation. MAST discharge 18416 has $I_{p}=950 \mathrm{kA}$, $B_{T}=0.4 \mathrm{~T}, \bar{n}_{e}=1.68 \times 10^{19} \mathrm{~m}^{-3}$, the safety factor at the magnetic axis predicted by the EFIT $\operatorname{code}^{27}$ is $q_{0}=0.78$ and $P_{\mathrm{NBI}}=3.32 \mathrm{MW}$. The rotation velocity at the magnetic axis was $0.19 v_{A}$ at the time the equilibrium was reconstructed $(0.318 \mathrm{~s})$. The electron temperature and density profiles were measured by the Thomson scattering diagnostic, while the current profile is found using the EFIT code. ${ }^{27}$ These profiles, together with the fixed boundary shape, are supplied to the HELENA code, ${ }^{25}$ which produces the equilibrium employed by MISHKA-F. ${ }^{6}$

The stability of the $n=1$ internal kink mode in the presence of strong toroidal rotation is also modeled consistently using the CASTOR-FLOW code. ${ }^{12}$ In order to do this, the freeboundary equilibrium code DIVA (Ref. 26) has been used to generate an equilibrium that includes the strong toroidal rotation. By increasing the toroidal rotation systematically in both the equilibrium calculation and the perturbation analysis, the toroidal flow required to marginally stabilize the $n=1$ internal kink mode can be calculated and compared to the result from the nonself-consistent MISHKA-F analysis. Figure 9 shows the equilibrium profiles for MAST discharge 18416 , while the stability of the $n=1$ internal kink mode modeled using both the consistent and perturbative codes is illustrated in Fig. 10. It is clear that for these equilibrium profiles, the toroidal rotation required to marginally stabilize 


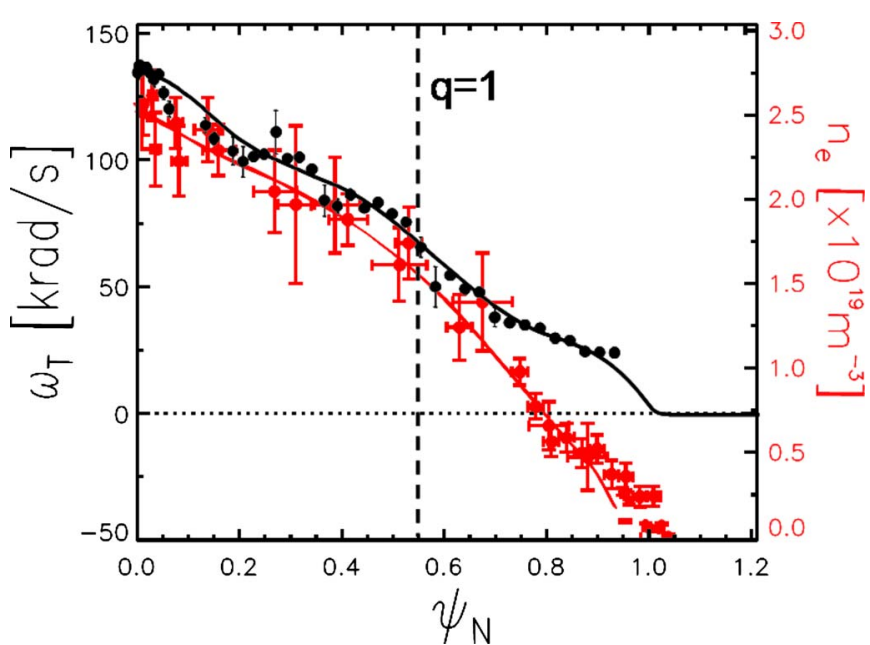

FIG. 9. (Color online) The density and rotation profiles in MAST discharge 18416 .

the kink mode is significantly different if the rotation is selfconsistently included in the equilibrium. Here we have chosen particular MAST profiles, which exemplify that the inclusion of toroidal flow in the equilibrium can have a significant effect. However, for more typical MAST profiles, the difference between the rotation required to marginally stabilize the internal kink mode as found using either a consistent or an inconsistent treatment of the equilibrium flow is not substantial, as discussed in Ref. 19. Furthermore, the inconsistent stability analysis always finds a larger critical rotation required for mode stability, meaning that it represents an upper bound on the marginal flow, partially justifying the exploitation of inconsistent numerical analyses in previous work. ${ }^{7}$

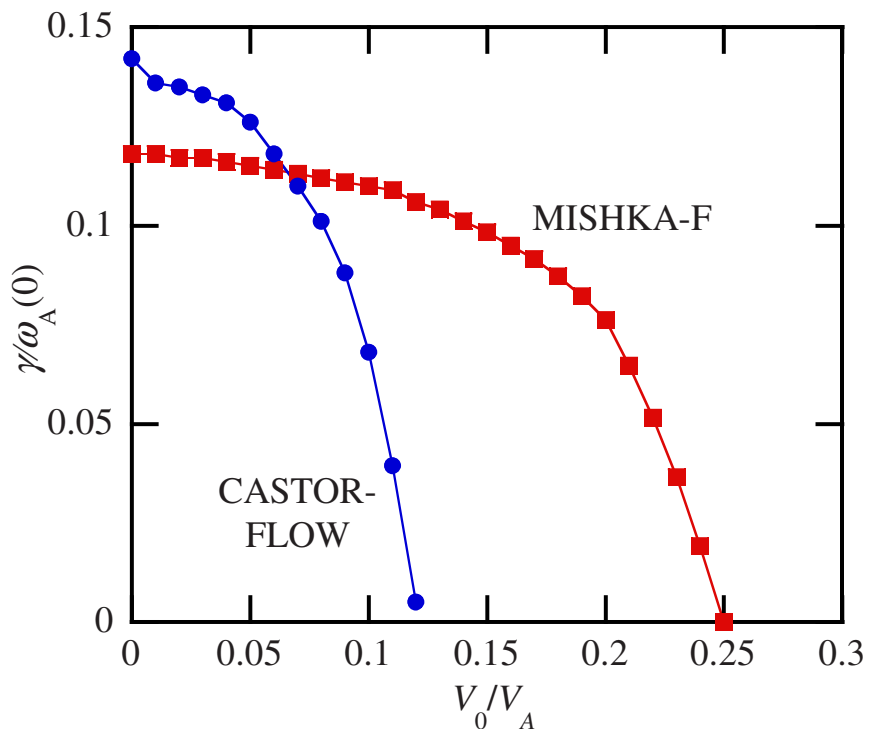

FIG. 10. (Color online) The effect of toroidal rotation on the $n=1$ internal kink mode growth rate in the MAST equilibrium in Fig. 9, calculated with either static (HELENA+MISHKA-F) or flowing (DIVA +CASTOR-FLOW) equilibria. It can be seen that including flow in the equilibrium has a significant effect on the stability boundary for this MAST plasma.

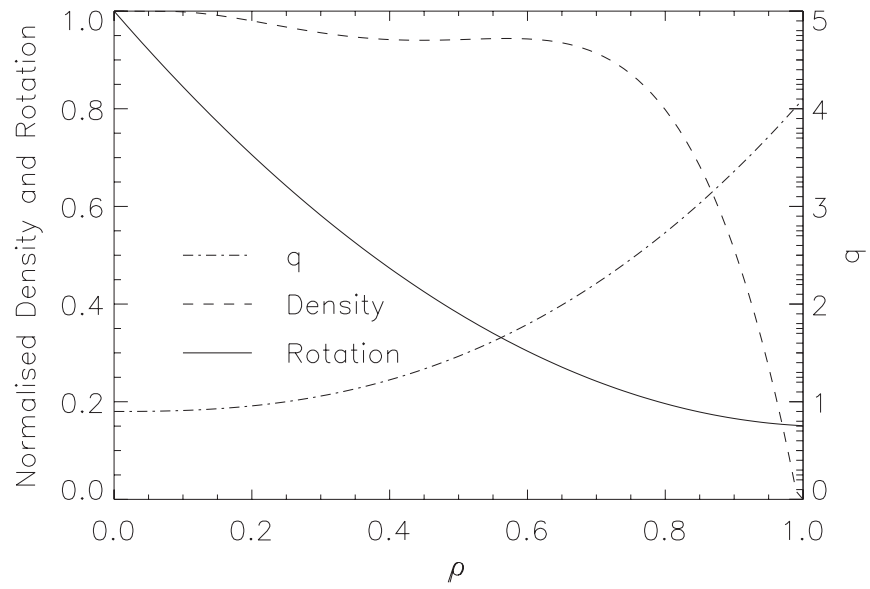

FIG. 11. Density, rotation, and $q$-profiles for a typical conventional aspect ratio tokamak plasma.

From Fig. 10 it is evident that the growth rate arising from the two calculations in the static case is slightly different. This is due to the difference between the free- and fixedboundary equilibrium solvers. The HELENA code is constrained to the plasma shape derived by EFIT, whereas the DIVA code takes as input the currents in the poloidal field coils and calculates the resultant plasma configuration. Consequently, there is a small difference in the plasma shape, which results in the difference in this static growth rate. Care was taken to match the static $q$-profiles, in particular the radial location of the $q=1$ surface, arising from the two equilibrium codes.

\section{B. Conventional aspect ratio analysis}

In order to analyze the effect of rotation on the stability of the $n=1$ internal kink mode for a conventional aspect-ratio plasma, both consistent and inconsistent stability analyses have been performed for such an equilibrium. The equilibrium profiles employed are illustrated in Fig. 11, and additional parameters of the equilibrium used are elongation $=1.71$, triangularity $=0.32$, and $\varepsilon_{a}=0.31$. The density profile used is similar to that given in Ref. 28 , the rotation profile is estimated from the profiles in Ref. 29, and the $q$-profile is taken from Ref. 30. Of course, these profiles are not intended to be self-consistent, since they are taken from different plasmas, but simply to be indicative of typical plasma profiles in a large aspect-ratio device. Figure 12 shows the growth rate of the internal kink mode as the rotation speed at the magnetic axis is varied. It is clear that treating the equilibrium flow consistently yields a very different effect of toroidal flow when compared to an inconsistent equilibrium treatment. Indeed, increasing toroidal flow is stabilizing in the consistent CASTOR-FLOW modeling, but results in a destabilization of the kink mode when modeled with the MISHKA-F code using an inconsistent equilibrium, similarly to the results obtained previously for the model equilibria in Figs. 1 and 6. The significant difference in kink mode stability illustrates how important the treatment of the equilibrium flow is for these typical profiles. 


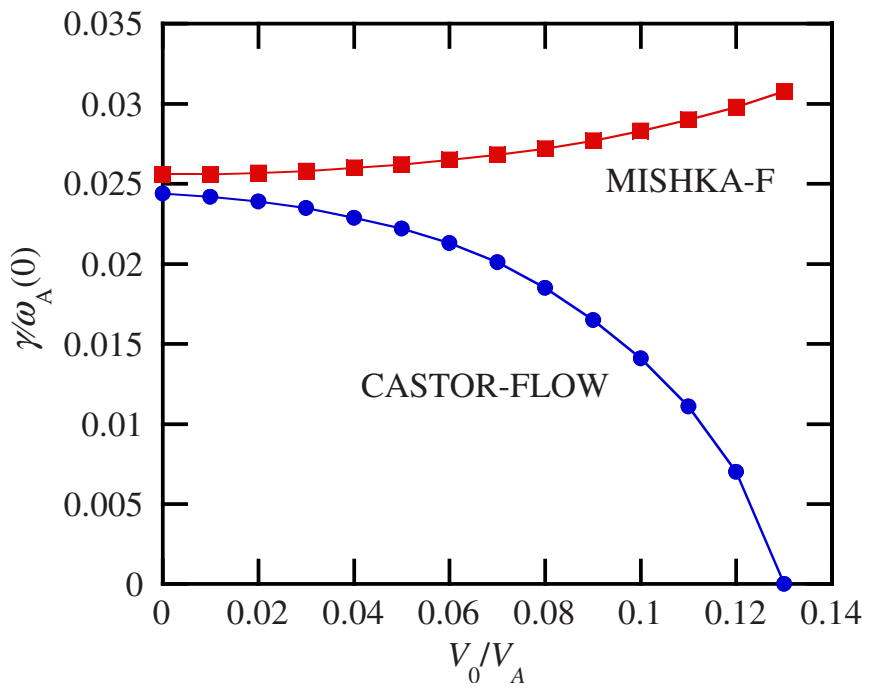

FIG. 12. (Color online) The effect of toroidal rotation on the $n=1$ internal kink mode growth rate for the equilibrium profiles shown in Fig. 11, calculated with both the static (MISHKA-F) and flowing (CASTOR-FLOW) treatments. It can be seen that including flow in the equilibrium has a significant effect on the stability boundary for this kind of equilibrium.

\section{SUMMARY AND CONCLUSIONS}

The effect of sonic, toroidal rotation on the ideal, internal kink instability with mode numbers $m=n=1$ in tokamak plasmas with $q<1$ in the core region has been investigated both analytically and numerically in this paper. The investigation has especially focused on the role that the centrifugal effects from the rotation on the plasma equilibrium play for the stability of this mode. Analytically, this has been done by repeating the stability analysis in Ref. 5 with the tags $\sigma_{1}$ and $\sigma_{2}$ included in the equilibrium profiles in Eq. (2a) and in the expression for the poloidal beta in Eq. (2b), respectively. Thus, $\sigma_{1}=\sigma_{2}=1$ corresponds to a fully self-consistent problem with the centrifugal effects from the rotation included in the equilibrium whereas a nonself-consistent problem where such effects are neglected is obtained for $\sigma_{1}=\sigma_{2}=0$. Results from this analytical model are thereafter compared with numerical results obtained by using two different MHD stability codes, viz. CASTOR-FLOW (Ref. 12) where centrifugal effects are included in the equilibrium and MISHKA-F (Ref. 6) where such effects are not included.

The main result of the paper is that, already for rather modest flow speeds, the centrifugal effects on the equilibrium are found to be of crucial importance for the stability of the $m=n=1$ mode in rotating plasmas, and if these effects are not taken into account, misleading or even erroneous results can be obtained. Such an example is shown in Fig. 1, which illustrates a case (with parabolic profiles of both the density and the rotation frequency) where the inconsistent model predicts an increasing growth rate with increasing plasma rotation whereas the mode is stabilized when $V_{0} / V_{A} \sim 0.005$ in the consistent model. In terms of the sonic Mach number $\mathcal{M}$, stabilization occurs for $\mathcal{M} \sim 0.05$ in this case. The good agreement between analytical and numerical results in this figure is due to the small value of $\varepsilon_{a}=0.1$ used, and validates the analytical model which is based on an ex- pansion in large aspect ratio, $\varepsilon \ll 1$. Increasing $\varepsilon$ to realistic values, a similar difference between the inconsistent and consistent models is found, but the agreement between analytical and numerical results is now more qualitative than quantitative (especially in the consistent case). This can be seen in Fig. 6, where the same situation as in Fig. 1 is shown at a larger value of $\varepsilon_{a}=0.3$. Also here, the inconsistent model predicts a destabilizing effect from the rotation (for parabolic density and rotation frequency profiles) whereas in the consistent model the kink mode is stabilized at flow speeds slightly exceeding $V_{0} / V_{A} \sim 0.03$, corresponding to $\mathcal{M} \sim 0.12\left(V_{0} / V_{A} \sim 0.02\right.$ and $\mathcal{M} \sim 0.08$ in the analytical model). Similar results can be found also in realistic equilibria, as shown in Fig. 12 where completely different internal $n=1$ kink growth rates as functions of rotation velocity are obtained from CASTOR-FLOW and MISHKA-F for a plasma equilibrium with the shape of the cross section and the profiles of plasma density, rotation velocity, and safety factor $q$ typical of a conventional aspect-ratio plasma.

From the analytical theory, the distinctly different properties of the consistent and inconsistent models can be explained by the finite frequency in Eq. (13b) (for $\sigma_{1}>0$ ) of the GAM induced by the plasma rotation. ${ }^{15}$ This frequency appears in the stability problem as a finite continuum frequency in the $q \approx 1$ layer, and it is this continuum frequency that, in a mathematical sense, is responsible for the stabilization seen in the examples discussed above. It is this stabilization that is interpreted as gyroscopic in Ref. 4 and explained in terms of the BV effect in Ref. 5. By the combined analytical and numerical study of the present paper, we have shown that this stabilization is entirely connected with the finite tangential gradients of the plasma density and plasma pressure that the centrifugal force creates on the flux surfaces, and does not occur as a consequence of the rotation alone if these gradients are not taken into account. This is consistent with the physical interpretation of the stabilization suggested in Ref. 5. It should be remarked, though, that the role of the parallel component of $\xi$ is slightly overemphasized in that paper. Similarly to the ordinary GAM,${ }^{16}$ the rotation-induced GAM has poloidal and toroidal components of $\xi$ of comparable magnitude. ${ }^{18}$

In addition to the stabilization by the coupling to the rotation-induced GAM, the radial profiles of the plasma density $\rho_{0}$ and rotation frequency $\Omega$ are also of importance for the stability of the kink mode. These profile effects can be expressed in terms of the contribution $\delta \hat{W}_{\text {Rot1 }}$ to the potential energy given by Eq. (31) and, in the special case of parabolic or flat profiles of both $\rho_{0}$ and $\Omega$, by Eq. (32). This result is identical to the contribution $W_{\text {Erot }}+W_{\text {flutter }}$ to the internal energy calculated in Ref. 4, and the same effect is accordingly included in the coefficient $r^{2} d A_{2} / d r$ of the inertia operator $\mathcal{T}_{1}$ derived in Ref. 5. Formally, this contribution to the potential energy of the internal kink instability is one order higher in $\varepsilon$ than the static part represented by $\delta \hat{W}_{\text {Bussac }}{ }^{11}$ In practice, however, and unless $\Delta q=1-q_{0}$ is large, these profile effects become significant at realistic values of $\varepsilon_{a}$, and their importance increases with decreasing aspect ratio. This is illustrated schematically in Fig. 7, where the critical rotation ve- 
locity for stabilization is shown as a function of $\varepsilon_{a}$ for different combinations of the profiles of $\rho_{0}$ and $\Omega$. In the limit $\varepsilon_{a} \rightarrow 0, \quad V_{0, \text { crit }} \sim \varepsilon_{a}^{3 / 2}$ independently of the profiles whereas already for $\varepsilon_{a} \sim 0.1-0.2$ or so the profile effects are seen to become important. From Eqs. (31) and (32) it is seen that a decreasing density with minor radius is destabilizing whereas a decreasing rotation velocity with minor radius is stabilizing. The most unstable combination in Fig. 7 is therefore a flat flow and a parabolic density profile, whereas the opposite combination gives the most stable situation. With an increasing value of $\Delta q$, however, the magnitude of $\delta \hat{W}_{\text {Bussac }}$ increases (since $\delta \hat{W}_{\text {Bussac }} \sim \Delta q$ when $\Delta q$ is small ${ }^{11,21,22}$ ) implying that the relative importance of $\delta \hat{W}_{\text {Rotl }}$, and thereby the profile effects included in this term, decreases. Instead, the effect of the profiles on the total $\beta_{p}$ in Eqs. (2b) and (27) becomes more important, and since especially the effect of a parabolic rotation profile is to increase the total $\beta_{p}$, for a sufficiently large $\Delta q$ the overall effect of such a rotation profile becomes destabilizing instead of the stabilizing effect it has in $\delta \hat{W}_{\text {Rot1 }}$. Such a case is illustrated in Fig. 8.

We emphasize that the profile effects expressed by $\delta \hat{W}_{\text {Rot1 }}$ in a rotating plasma do not require that the centrifugal effects are included in the equilibrium. These effects are therefore seen also in the present as well as previous computations using the MISHKA-F code. For profiles of the density and rotation such that this rotational effect is stabilizing, the behavior of the growth rate as a function of rotation velocity can therefore be quite similar in the inconsistent and consistent models, see e.g., Figs. 4, 5, and 10. This is the case also for the equilibria where rotational stabilization is seen in several previous studies using MISHKA-F. ${ }^{6-8}$ Examples of where the rotational stabilization in the inconsistent and consistent cases indeed look very similar are given in a related paper, ${ }^{19}$ where the profile effects on the internal $n=1$ instability, with particular application to MAST, are studied in more detail.

Finally, we also mention that a great deal of work has been done in the past on toroidal flow and flow shear effects on the MHD stability of other modes, especially resistive wall modes, ${ }^{31-36}$ ballooning modes, ${ }^{37-45}$ the quasiinterchange mode, ${ }^{46,47}$ and Mercier modes. ${ }^{24,48}$ For the quasiinterchange mode, stabilization by the second GAM frequency both in the case of rigid rotation and sheared rotation were discussed in Refs. 46 and 47, respectively. In the latter case, the profile effects from the rotation described by the $r^{2} d A_{2} / d r$ coefficient in the inertia operator were neglected, but the destabilizing effect from the enhanced $\beta_{p}$ due to the flow shear was taken into account. In the case of Mercier modes, stabilization by the finite GAM (or BV) frequency in rotating plasmas was shown in Ref. 24. A similar analysis had previously been made by Zheng et al., ${ }^{48}$ but since this analysis assumed $\Gamma=1$, the stabilizing effect from the lowfrequency GAM is not taken into account in that work. With the exception of Refs. 4, 5, 24, 46, and 47 and the stability analysis of ballooning modes in rotating plasmas by Grassie and Krech, ${ }^{44,45}$ the effects of an equilibrium modified by the centrifugal force, studied in the present paper, do not seem to have been discussed previously in the literature. In Ref. 45, however, stabilizing effects of both rigid and sheared toroidal rotations somewhat similar to the results obtained in the present paper were found. Especially, the reduction of the growth rate of the ballooning mode with increasing rigid rotation found by Grassie and Krech was interpreted by these authors, at least partly, in terms of the modified pressure distribution on the magnetic surfaces induced by the rotation, i.e., a stabilizing effect strongly resembling the stabilization by the second GAM frequency discussed in the present paper. Concerning the stabilizing effect of velocity shear on the ballooning modes discussed in Ref. 45 as well as in other, related studies, ${ }^{37-43}$ we point out that corrections of the growth rate due to rotation shear that do not depend on the sign of $\Omega^{\prime}$, e.g., the growth rate in Eq. (16) in Ref. 39, must have a different origin than the mechanism underlying $\delta \hat{W}_{\text {Rot1 }}$, since the sign of $\Omega^{\prime}$ is very important in this term. Hence, there seems to be several different effects that rotation and rotation shear can have on the MHD stability of tokamak plasmas, and the area as a whole is obviously quite complex and needs further investigation. Another important area is to include kinetic effects in the analysis of the centrifugal effects on the plasma stability. Such an extension is particularly important in view of the dependence of the stabilizing, second GAM frequency in Eq. (13b) on plasma compressibility and $\Gamma$.

\section{ACKNOWLEDGMENTS}

The authors would like to thank Dr. E. Strumberger, Dr. H. P. Zehrfeld, Dr. C. Konz, and their co-workers at IPP Garching for providing us with the CASTOR-FLOW and DIVA codes. This work was partly funded by the United Kingdom Engineering and Physical Sciences Research Council and by the European Communities under the contracts of Association between EURATOM, the Swedish Research Council (VR), UKAEA, and the Fonds National Suisse de la Recherche Scientifique. The views and opinions expressed herein do not necessarily reflect those of the European Commission.

${ }^{1}$ S. von Goeler, W. Stodiek, and N. Sauthoff, Phys. Rev. Lett. 33, 1201 (1974).

${ }^{2}$ B. Lloyd, R. J. Akers, F. Alladio, Y. Andrew, L. C. Appel, D. Applegate, K. B. Axon, N. Ben Ayed, C. Bunting, R. J. Buttery, P. G. Carolan, I. Chapman, D. Ciric, J. W. Connor, N. J. Conway, M. Cox, G. F. Counsell, G. Cunningham, A. Darke, E. Delchambre, R. O. Dendy, J. Dowling, B. Dudson, M. Dunstan, A. R. Field, A. Foster, S. Gee, L. Garzotti, M. P. Gryaznevich, A. Gurchenko, E. Gusakov, N. C. Hawkes, P. Helander, T. C. Hender, B. Hnat, D. F. Howell, N. Joiner, D. Keeling, A. Kirk, B. Koch, M. Kuldkepp, S. Lisgo, F. Lott, G. P. Maddison, R. Maingi, A. Mancuso, S. J. Manhood, R. Martin, G. J. McArdle, J. McCone, H. Meyer, P. Micozzi, A. W. Morris, D. G. Muir, M. Nelson, M. R. O'Brien, A. Patel, S. Pinches, J. Preinhaelter, M. N. Price, E. Rachlew, C. M. Roach, V. Rozhansky, S. Saarelma, A. Saveliev, R. Scannell, S. E. Sharapov, V. Shevchenko, S. Shibaev, K. Stammers, J. Storrs, A. Surkov, A. Sykes, S. Tallents, D. Taylor, N. Thomas-Davies, M. R. Turnyanskiy, J. Urban, M. Valovic, R. G. L. Vann, F. Volpe, G. Voss, M. J. Walsh, S. E. V. Warder, R. Watkins, H. R. Wilson, M. Wisse, and the MAST and NBI Teams, Nucl. Fusion 47, S658 (2007).

${ }^{3}$ J. E. Menard, R. E. Bell, E. D. Fredrickson, D. A. Gates, S. M. Kaye, B. P. LeBlanc, R. Maingi, S. S. Medley, W. Park, S. A. Sabbagh, A. Sontag, D. Stutman, K. Tritz, W. Zhu, and the NSTX Research Team Nucl. Fusion 45, 539 (2005).

${ }^{4}$ F. L. Waelbroeck, Phys. Plasmas 3, 1047 (1996). 
${ }^{5}$ C. Wahlberg and A. Bondeson, Phys. Plasmas 7, 923 (2000).

${ }^{6}$ I. T. Chapman, S. E. Sharapov, G. T. A. Huysmans, and A. B. Mikhailovskii, Phys. Plasmas 13, 062511 (2006).

${ }^{7}$ I. T. Chapman, T. C. Hender, S. Saarelma, S. E. Sharapov, R. J. Akers, N. J. Conway, and MAST Team, Nucl. Fusion 46, 1009 (2006).

${ }^{8}$ I. T. Chapman, S. D. Pinches, J. P. Graves, R. J. Akers, L. C. Appel, R. V. Budny, S. Coda, N. J. Conway, M. de Bock, L.-G. Eriksson, R. J. Hastie, T. C. Hender, G. T. A. Huysmans, T. Johnson, H. R. Koslowski, A. Krämer-Flecken, M. Lennholm, Y. Liang, S. Saarelma, S. E. Sharapov,I. Voitsekhovitch, the MAST and TEXTOR Teams, and JET-EFDA Contributors, Plasma Phys. Controlled Fusion 49, B385 (2007).

${ }^{9}$ J. P. Graves, C. Angioni, R. V. Budny, R. J. Buttery, S. Coda, L.-G. Eriksson, C. G. Gimblett, T. P. Goodman, R. J. Hastie, M. A. Henderson, H. R. Koslowski, M. J. Mantsinen, An. Martynov, M.-L. Mayoral, A. Mück, M. F. F. Nave, O. Sauter, E. Westerhof, and JET-EFDA Contributors, Plasma Phys. Controlled Fusion 47, B121 (2005).

${ }^{10}$ E. R. Priest, Solar Magnetohydrodynamics (Reidel, Dordrecht, 1982), Chap. 4.

${ }^{11}$ M. N. Bussac, R. Pellat, D. Edery, and J. L. Soule, Phys. Rev. Lett. 35, 1638 (1975).

${ }^{12}$ E. Strumberger, S. Günter, P. Merkel, S. Riondato, E. Schwarz, C. Tichmann, and H. P. Zehrfeld, Nucl. Fusion 45, 1156 (2005).

${ }^{13}$ W. Kerner, S. Poedts, J. P. Goedbloed, G. T. A. Huysmans, B. Keegan, and E. Schwarz, Proceedings of the 18th EPS Conference on Controlled Fusion and Plasma Physics (Berlin) (EPS, Geneva, 1991), Vol. 4, p. 89.

${ }^{14}$ G. T. A. Huysmans, J. P. Goedbloed, and W. Kerner, Phys. Fluids B 5, 1545 (1993).

${ }^{15}$ C. Wahlberg, Phys. Rev. Lett. 101, 115003 (2008).

${ }^{16}$ N. Winsor, J. L. Johnson, and J. M. Dawson, Phys. Fluids 11, 2448 (1968).

${ }^{17}$ B. van der Holst, A. J. C. Belien, and J. P. Goedbloed, Phys. Plasmas 7, 4208 (2000).

${ }^{18}$ C. Wahlberg, Plasma Phys. Controlled Fusion 51, 085006 (2009).

${ }^{19}$ I. T. Chapman, J. P. Graves, C. Wahlberg, and the MAST Team, "The effect of plasma profile variation on the stability of the $n=1$ internal kink mode in rotating tokamak plasmas," Nucl. Fusion (submitted).

${ }^{20}$ E. Frieman and M. Rotenberg, Rev. Mod. Phys. 32, 898 (1960).

${ }^{21}$ J. W. Connor and R. J. Hastie, "The effect of shaped plasma cross sections on the ideal internal kink mode in a tokamak," Report No. CLM-M-106, Culham Laboratory, Abingdon, Oxfordshire, 1985; AIP document No. PAPS PFLDA-30-1756-36.

${ }^{22}$ J. P. Graves and C. Wahlberg, Phys. Plasmas 14, 082504 (2007).

${ }^{23}$ C. Wahlberg and A. Bondeson, Phys. Lett. A 271, 285 (2000).

${ }^{24}$ C. Wahlberg and A. Bondeson, Phys. Plasmas 8, 3595 (2001).

${ }^{25}$ G. Huysmans, J. P. Goedbloed, and W. Kerner, Proceedings of the CP90 Conference on Computational Physics (World Scientific, Singapore, 1991), p. 371.
${ }^{26}$ H. P. Zehrfeld, 26th EPS Conference on Controlled Fusion and Plasma Physics (European Physical Society, Maastricht, 1999), Vol. 23J, p. 1421.

${ }^{27}$ L. L. Lao, J. R. Ferron, R. J. Groebner, W. Howl, H. St. John, E. J. Strait, and T. S. Taylor, Nucl. Fusion 30, 1035 (1990).

${ }^{28}$ J. Stober, O. Gruber, M. Kaufmann, R. Neu, F. Ryter, and W. Sandmann, H. Zohm, and the ASDEX Upgrade Team, Plasma Phys. Controlled Fusion 44, A159 (2002).

${ }^{29}$ D. Nishijima, A. Kallenbach, S. Günter, M. Kaufmann, K. Lackner, C. F. Maggi, A. Peeters, G. Pereverzev, B. Zaniol, and the ASDEX Upgrade Team, 31st EPS Plasma Physics Conference (European Physical Society, London, 2004), Paper No. P4.121.

${ }^{30}$ A. C. C. Sips, J. Stober, M. Reich, C. Forrest, O.Gruber, J. Hobirk, L. D. Horton, C. F. Maggi, P. McCarthy, M. Maraschek, V. Mertens, A. Stäbler, G. Tardini, and the ASDEX Upgrade Team, 33rd EPS Plasma Physics Conference (European Physical Society, Rome, 2006), Paper No. P2.130.

${ }^{31}$ A. C. Sontag, S. A. Sabbagh, W. Zhu, J. M. Bialek, J. E. Menard, D. A. Gates, A. H. Glasser, R. E. Bell, B. P. LeBlanc, M. G. Bell, A. Bondeson, J. D. Callen, M. S. Chu, C. C. Hegna, S. M. Kaye, L. L. Lao, Y. Liu, R. Maingi, D. Mueller, K. C. Shaing, D. Stutman, and K. Tritz, Phys. Plasmas 12, 056112 (2005).

${ }^{32}$ C. Gimblett, Nucl. Fusion 26, 617 (1986).

${ }^{33}$ M. S. Chu, J. M. Greene, T. H. Jensen, R. L. Miller, A. Bondeson, R. W. Johnson, and M. E. Mauel, Phys. Plasmas 2, 2236 (1995).

${ }^{34}$ A. Bondeson and D. J. Ward, Phys. Rev. Lett. 72, 2709 (1994).

${ }^{35}$ Y. Q. Liu, A. Bondeson, Y. Gribov, and A. Polevoi, Nucl. Fusion 44, 232 (2004).

${ }^{36}$ A. M. Garofalo, T. H. Jensen, L. C. Johnson, R. J. La Haye, G. A. Navratil, M. Okabayashi, J. T. Scoville, E. J. Strait, D. R. Baker, J. Bialek, M. S. Chu, J. R. Ferron, J. Jayakumar, L. L. Lao, M. A. Makowski, H. Reimerdes, T. S. Taylor, A. D. Turnbull, M. R. Wade, and S. K. Wong, Phys. Plasmas 9, 1997 (2002).

${ }^{37}$ W. A. Cooper, Plasma Phys. Controlled Fusion 30, 1805 (1988).

${ }^{38}$ R. Miller, F. Waelbroeck, A. Hassam, and R. Waltz, Phys. Plasmas 2, 3676 (1995).

${ }^{39}$ J. C. Connor, R. J. Hastie, and J. B. Taylor, Plasma Phys. Controlled Fusion 46, B1 (2004).

${ }^{40}$ H. R. Wilson, Plasma Phys. Controlled Fusion 35, 885 (1993).

${ }^{41}$ M. Furukawa and S. Tokuda, Nucl. Fusion 45, 377 (2005).

${ }^{42}$ F. L. Waelbroeck and L. Chen, Phys. Fluids B3, 601 (1991).

${ }^{43}$ J. B. Taylor, Phys. Plasmas 6, 2425 (1999).

${ }^{44}$ K. Grassie and M. Krech, Phys. Fluids B 2, 536 (1990).

${ }^{45}$ K. Grassie and M. Krech, Phys. Fluids B 2, 1864 (1990).

${ }^{46}$ C. Wahlberg, Plasma Phys. Controlled Fusion 47, 757 (2005).

${ }^{47}$ C. Wahlberg, 32nd EPS Conference on Plasma Physics (European Physical Society, Tarragona, 2005), Paper No. P4.068.

${ }^{48}$ L.-J. Zheng, M. S. Chu, and L. Chen, Phys. Plasmas 6, 1217 (1999). 